\title{
Incomplete Information and the Liquidity Premium Puzzle*
}

\author{
Yingshan Chen ${ }^{\dagger}$ \\ Min Dai $\ddagger$ \\ Luis Goncalves-Pinto § \\ Jing $\mathrm{Xu} \|$ \\ Cheng Yan **
}

May 2, 2020

\begin{abstract}
We examine the problem of an investor who trades in a market with unobservable regime shifts. The investor learns from past prices and is subject to transaction costs. Our model generates significantly larger liquidity premia compared to a benchmark model with observable market shifts. The larger premia are driven primarily by suboptimal risk exposure, as turnover is lower under incomplete information. In contrast, the benchmark model produces (mechanically) high turnover and heavy trading costs. We provide empirical support for the amplification effect of incomplete information on the relation between trading costs and future stock returns. We also show empirically that such amplification is not driven by turnover. Overall, our results can help explain the large disconnect between theory and evidence regarding the magnitude of liquidity premia, which has been a longstanding puzzle in the literature.
\end{abstract}

Keywords: Regime Shifts, Incomplete Information, Transaction Costs, Liquidity Premia.

JEL Classification: C61, D11, D91, G11.

\footnotetext{
* We appreciate the comments from the Editor, an Associate Editor, two anonymous referees, Hong Liu, Stavros Panageas, Yizhou Xiao, and participants at the Asian Quantitative Finance Conference, the Australasian Finance and Banking Conference, and the Bachelier Finance Society World Congress.

$\dagger$ South China University of Technology, Building 4, Wushan Road, Tianhe district, Guangzhou; Tel. +86 020-2223-6162; Email: mayshchen@scut.edu.cn

¥ National University of Singapore, 10 Lower Kent Ridge Road, S17-08-20, Singapore 119076; Tel. +65 6516-2754; Email: matdm@nus.edu.sg

$\S$ University of New South Wales, School of Banking and Finance, Sydney, NSW 2052, Australia; Tel. +612 9385 4291 ; Email: l.goncalves-pinto@unsw.edu.au

ฯ Chinese University of Hong Kong, Department of Finance, Hong Kong; Tel. (+852) 3943-7805; Email: lgoncalv@cuhk.edu.hk

I Renmin University of China, School of Finance, Mingde Main Building, 59 Zhongguancun Street, Beijing, China 100872, Tel. +86-010-8250-9260; Email: jing.xu@ruc.edu.cn

** University of Essex, Essex Business School, Colchester, CO4 3SQ, United Kingdom. Email: cheng.yan.1@cass.city.ac.uk
} 


\title{
Incomplete Information and the Liquidity Premium Puzzle
}

\begin{abstract}
We examine the problem of an investor who trades in a market with unobservable regime shifts. The investor learns from past prices and is subject to transaction costs. Our model generates significantly larger liquidity premia compared to a benchmark model with observable market shifts. The larger premia are driven primarily by suboptimal risk exposure, as turnover is lower under incomplete information. In contrast, the benchmark model produces (mechanically) high turnover and heavy trading costs. We provide empirical support for the amplification effect of incomplete information on the relation between trading costs and future stock returns. We also show empirically that such amplification is not driven by turnover. Overall, our results can help explain the large disconnect between theory and evidence regarding the magnitude of liquidity premia, which has been a longstanding puzzle in the literature.
\end{abstract}




\section{Introduction}

If investors could have access to complete information about the stock market shifts, they would be able to perfectly time such shifts and improve significantly their investment performance. However, this is generally not the case for individual investors, and it is also not true for investors that are considered to be more sophisticated. ${ }^{1}$ Therefore, given that information is typically incomplete, investors are likely to stay overinvested when the stock market switches from a bull to a bear state, and remain underinvested for the reverse switch. Naturally, these suboptimal portfolio allocations in turn translate into losses.

We argue that the suboptimal risk exposure imposed by incomplete information is likely to worsen in the presence of transaction costs. This in turn results in two important sources of utility losses for investors: (i) the trading cost charges associated with portfolio rebalancing, and (ii) the suboptimal risk exposure chosen to control the trading costs. Therefore, investors should demand additional returns to compensate for both (i) and (ii). From the outset, it is intuitive that (i) should play a more important role when information is complete, because in this case portfolio turnover is deemed to increase. In the presence of incomplete information, we should expect (ii) to be the primary driver of utility losses, because portfolio turnover should not be higher in this case. However, it is not obvious which one of these two settings should generate larger losses overall. In this paper, we present both theoretical and empirical analyses to address this tension. More importantly, given that the incomplete information case is likely to be more realistic, this paper is set out to understand if this new ingredient can help further explain the disconnect between theory and evidence regarding the magnitude of liquidity premia, which has been a longstanding puzzle in the literature.

The liquidity premium puzzle was first highlighted in the seminal work of Constantinides (1986). It shows that in the presence of transaction costs, continuous and unlimited trading (like in Merton $(1969,1971))$ are no longer possible, and even a tiny transaction charge can dramatically reduce the frequency and volume of trading for investors. More importantly, it shows that the liquidity premium (i.e., the maximum stock return the investor is willing to give up to reduce transaction costs to zero) is surprisingly small relative to the transaction cost rate. It shows that, the model-

\footnotetext{
${ }^{1}$ A main reference on the poor market-timing ability of individual investors is Barber, Lee, Liu, and Odean (2009). For evidence on the poor timing ability of institutional investors, see Graham and Harvey (1996), Daniel, Grinblatt, Titman, and Wermers (1997), Wermers (2000), Kacperczyk and Seru (2007), and Kacperczyk, van Nieuwerburgh, and Veldkamp (2014). For evidence related with financial advisors, see Linnainmaa, Melzer, and Previtero (2018).
} 
implied liquidity premium to transaction cost (LPTC) ratio is only about 0.07 , for a proportional transaction cost rate of $1 \%$. The author concludes that transaction costs only have a second-order effect on liquidity premia.

However, this conclusion is not in line with many empirical findings that suggest that transaction costs significantly influence the time-series and the cross-section of stock returns. For example, Amihud and Mendelson (1986) find that the LPTC ratio is about 1.90 for NYSE stocks.

The large discrepancy between theoretical and empirical findings is puzzling. In an attempt to solve this puzzle, Jang, Koo, Liu, and Loewenstein (2007) (JKLL henceforth) argue that the findings in Constantinides (1986) depend on the crucial assumption of a constant investment opportunity set. They show that transaction costs can have a significantly larger effect on liquidity premia when it is assumed that the investment opportunity set of the investor varies over time. This is a very intuitive argument, because time-variation in investment opportunities exogenously induces investors to trade more frequently, which creates a much heavier trading cost charge, and investors demand compensation for such additional charges. However, the LPTC ratio that they find, for a reasonable calibration of their regime-switching model, is about 0.50 . Compared to the empirical evidence, this is not large enough to fully explain the liquidity premium puzzle.

We build on the model of JKLL to allow for a more realistic assumption regarding the information available to the investors. They have assumed that stock market regime shifts are perfectly observable. This induces investors to immediately rebalance their portfolio at the switching times to the optimal policy under the new regime, which mechanically generates a heavy trading cost charge. We relax their assumption of perfect observability of regime shifts. As discussed above, it is often the case in practice that investors only realize that they are in a bear or a bull market when it is already rather late for them to adjust their portfolios. We therefore assume that investors cannot observe the regime shifts but can calculate the probability that they are living in a given regime from observing past movements in stock prices.

In our model, the optimal policy of the investor depends not only on the current stock price, but also on the performance of the stock over a certain lookback period. The intuition is as follows: the investor needs to observe the time-series of stock prices to learn about the market regime she is living in, and she updates her portfolio according to this learning process. We synthesize in a single measure the information provided by the time-series of past stock prices. Specifically, this measure captures the likelihood that the investor is living in a bull market. Intuitively, when stock prices go up for a certain period of time, it is more likely that our investor is living in a bull 
market. If instead prices have been coming down for a certain period of time, then it is less likely that the investor is living in a bull market. As a result, the investor initiates, holds, or liquidates positions in her portfolio based on her estimation of this likelihood. This is similar to the posterior distribution in Bayesian updating. This posterior can take many different values within the interval between zero and one, which means that the investor only gradually adjusts her portfolio to the conditions of the upcoming stock market regime. Therefore, she is expected to remain overinvested (underinvested) in stock when the regime switches from bull to bear (from bear to bull). The slow adjustments in stock positions make the investor's portfolio deviate significantly from what would be optimal if she could perfectly observe the regime shifts. The suboptimal risk exposure, induced by a combination of incomplete information and transaction costs, then translates into heavy utility losses for investors. As a result, investors should demand high future stock returns (i.e., high liquidity premia) to compensate for such losses.

We allow for differences in mean returns between bull and bear regimes, but we set the volatilities in the two regimes to be equal. This guarantees that the market regimes are indeed unobservable. If return volatilities were different in bull and bear regimes, the investor could perfectly estimate the regime she is living in by using the quadratic variation in stock prices. ${ }^{2}$ We use the point estimates of average return for bull and bear regimes calculated for the U.S. market by Ang and Bekaert (2002). ${ }^{3}$ We also use their results to calibrate the duration of bull and bear markets in our model. They estimate that the average duration of a bull market is about four years and a quarter, and the average duration of a bear market is only seven months. Therefore, in statistical terms, the bear market only has a small effect on the overall market performance. However, we show that such low-frequency and low-duration events can play an economically important role in the portfolio decisions of investors.

We find the magnitude of the liquidity premia to increase significantly under incomplete information. For instance, using a (round-trip) transaction cost rate of 1.4\% (the average bid-ask spread

\footnotetext{
${ }^{2}$ Our assumption that regimes differ only in the drift of the stock price process is similar to the models of Honda (2003) and Liu (2011). This assumption allows us to reformulate our problem as a singular stochastic control problem with complete information using the filtering technique proposed in Wonham (1965).

3 They estimate a two-regime Markov switching model, and report that the mean log return is $1.2829 \%$ per month in the bull regime and $-1.2881 \%$ per month in the bear regime. They show that the difference between these point estimates is economically large, but they only find weak evidence that these two means are statistically different. This is intuitive, because bear regimes are typically shorter than bull regimes, which leads to a much lower number of observations for the bear markets, and larger standard errors. We abstract from these statistical properties of the market regimes, and focus on the impact that low-frequency and low-duration bear markets can have on the portfolio decisions of investors.
} 
of the sample used in Amihud and Mendelson (1986)), the LPTC ratio under complete information is 0.64 , but under incomplete information it is $90 \%$ higher, at $1.22 .{ }^{4}$ We conclude that one should account for the effects of incomplete information to further bridge the gap between theory and the empirical findings on the magnitude of liquidity premia. ${ }^{5}$

In general, transaction costs decrease an investor's utility through two channels: (i) the wealth that is consumed by the transaction costs paid in connection to the formation, rebalancing, and liquidation of the portfolio, and/or (ii) the suboptimal risk exposure due to the no-trade region created by transaction costs. We show that the main source of the extra liquidity premia generated by our model is the suboptimal risk exposure chosen by the investor to control the transaction costs. There are two mechanisms at work. First, when the investor only has access to incomplete information about market shifts, she needs to be constantly updating her estimate of the probability of living in a bull regime so as to decide on whether she should rebalance her portfolio or not. From the investor's perspective, the investment opportunity set is constantly changing. ${ }^{6}$

Second, uncertainty about the state of the market regime leads the investor to refrain from trading on her (potentially misestimated) probabilities of being in a given regime. In particular, the interaction between incomplete information and trading costs leads to a much wider no-trading region in the optimal policy. This decreases the frequency with which the investor trades, pushing her investment policy further away from the zero-transaction-cost optimum. This is in contrast with the results in JKLL, which are driven mostly by the increase in the amount and frequency of trading induced (mechanically) by the fully observable regime shifts. ${ }^{7}$

\footnotetext{
${ }^{4}$ It is important to highlight that our benchmark model is not exactly the same as in JKLL. This is not only because we use a different calibration, but also because the investment horizon in our model is finite. Thus, the LPTC ratio in the case with complete information does not match the ratio found in their paper.

${ }^{5}$ Dai, Li, Liu, and Wang (2016) examine the problem of an investor who trades in a market only when it is open for trading. They show that, the introduction of market closures, and accounting for the differences in return volatilities between trading and non-trading periods, can generate LPTC ratios with a magnitude that is in line with existing empirical studies. We propose an alternative mechanism to explain the liquidity premium puzzle. The model in Dai, Li, Liu, and Wang (2016) is effectively a regime-switching model in which regimes switch at high frequency (e.g., daily), are deterministic, and are fully observable. We consider a model in which regime shifts occur at a much lower frequency (e.g., once every four years) and are not observable.

${ }^{6}$ In our model, the true process driving stock prices is distinct from the process perceived by the investor. This is fundamentally different from a model with a stochastic stock price drift that is fully observable. In such a model, the true and perceived processes that drive stock prices are exactly the same. We have solved a model with complete information and a stochastic (slow moving) investment opportunity set, and we did not find the amplification effect on the relation between trading costs and liquidity premia that we document in this paper. The results of this alternative model are presented in Appendix A.4.

${ }^{7}$ For our baseline parameter values, we show that the discounted value of the transaction costs (PVTC) in the incomplete information model is only $40 \%$ of that in the complete information model, but it is more than $50 \%$ larger if compared to the model with constant investment opportunities of Constantinides (1986).
} 
In the empirical section of this paper, we provide evidence consistent with the novel implications of our theory model. In particular, we test two hypotheses: (i) information uncertainty amplifies the relation between trading costs and future stock returns, and (ii) stock turnover is lower under incomplete information, which means that it is not the main driver of liquidity premia.

We use the methodology and measures proposed in Hasbrouck (2009), which documents a positive relation between trading costs and future stock returns. To assess the impact of information uncertainty on the magnitude of this relation, we create an interaction between the trading cost measure and an indicator variable for values of macro uncertainty above the historical median. We use three sets of macro uncertainty proxies: (i) the three-component economic policy uncertainty (EPU) measure, and the news-based measure of economic policy uncertainty (NEPU) from Baker, Bloom, and Davis (2016); (ii) the 12-month financial uncertainty (FINU) index, the 12-month macro uncertainty (MACU) index, and the 12-month real uncertainty (REAU) index from Jurado, Ludvigson, and Ng (2015); and (iii) the CBOE VIX index.

We form one hundred portfolios by sequentially sorting stocks based on the market beta and the effective trading cost as of December of the prior year. These two sorting variables are estimated following Hasbrouck (2009). The dependent variable is the monthly excess return (relative to the risk-free rate) of each portfolio in the year after portfolio formation. The above/below-median uncertainty indicators are also measured as of December of the prior year, and the medians are calculated using the values of the uncertainty proxies in the 36 -month period prior to December. Our final sample covers the period 1994-2017.

We find a significantly stronger positive relation between trading costs and future stock returns during periods with uncertainty above the median. For instance, for a $1 \%$ increase in the effective trading cost, stocks provide an additional $0.19 \%$ monthly excess return (or $2.28 \%$ annualized) during periods defined ex-ante as having above-median EPU, compared to periods with below-median EPU. If instead we use NEPU as the proxy for uncertainty, the additional monthly excess return for periods with above-median versus below-median uncertainty is $0.51 \%$ (or $6.12 \%$ annualized), for a $1 \%$ increase in effective trading cost. The difference is even larger when using VIX as the uncertainty proxy, at about $0.53 \%$ monthly (or $6.36 \%$ annualized). If we use the uncertainty indices from Jurado, Ludvigson, and Ng (2015), the differences in future monthly excess returns between above- and below-median uncertainty periods are $0.32 \%$ for FINU (3.84\% annualized), $0.49 \%$ for MACU (5.88\% annualized), and $0.30 \%$ for REAU (3.6\% annualized).

Overall, these results are consistent with our theory that argues that information uncertainty 
has an important amplification effect on the relation between trading costs and future stock returns.

Regarding the mechanism, liquidity premia can be driven not only by the trading cost charge itself, which is a function of stock turnover, but also by the suboptimal risk exposure chosen to control the trading costs, which does not depend on turnover. In our theory section, we argue that the second channel predominates. To test this implication, we regress future portfolio turnover on the interaction between the trading cost measure and the above/below-median uncertainty indicators. Overall, the trading cost is always negatively related with turnover. We find that, for four out of the six uncertainty proxies that we use, portfolio turnover is significantly lower in periods with above-median uncertainty. Within the four uncertainty proxies with significant effects, EPU appears to be the strongest, with portfolio turnover being $95 \%$ lower in periods with above-median EPU, compared to periods with below-median EPU. The weakest is REAU with portfolio turnover being $22 \%$ lower in periods with above-median REAU compared to periods with below-median REAU.

These results suggest that the main mechanism driving the higher liquidity premia in periods of high uncertainty is not the trading cost charges associated with portfolio turnover, but the suboptimal risk exposure chosen to control the trading costs, as predicted by our theory model.

The rest of our paper is organized as follows. In Section 2, we provide a brief review of the related research. In Section 3, we present the theoretical framework and describe its main assumptions. Section 4 describes the optimal investment policies and the liquidity premia results. We provide empirical support for our model in Section 5. We conclude in Section 6, and provide extra analyses and proofs of the analytical results in the Appendix.

\section{Related Literature}

A long body of research has been conducted on the optimal portfolio choice in the presence of transaction costs, starting with the seminal work of Constantinides (1986), and including Davis

and Norman (1990), Shreve and Soner (1994), Liu and Loewenstein (2002), Liu (2004), Lynch and Tan (2010), Dai, Jin, and Liu (2011), among many others.

Constantinides (1986) first highlighted the theoretical second-order effect of transaction costs on liquidity premia, which was puzzling given the first-order effect found in empirical research. Recent research has been trying to resolve this discrepancy. To the best of our knowledge, there are three main references on this topic. The references are JKLL, Lynch and Tan (2011), and 
Dai, Li, Liu, and Wang (2016). The common element in these three references is the idea that one should allow for time-varying investment opportunities. They all show that this feature is crucial to generate a theoretical first-order effect of trading costs on liquidity premia, and that the conclusion of Constantinides (1986) is driven by the assumption of a constant investment opportunity set.

In the introductory section, we have described extensively the model in JKLL, which offers the benchmark model on which we build the theoretical analysis of our paper. Lynch and Tan (2011) use a discrete-time framework and incorporate return predictability, labour income, and statedependent transaction costs. However, after reasonable calibration, their result is still significantly smaller than what is documented in empirical evidence. Dai, Li, Liu, and Wang (2016) show that, if one incorporates in the model the well-established fact that market volatility during trading periods (i.e., from open to close on the same trading day) is significantly larger than the volatility during non-trading periods (from close in one day to open in the following trading day), then transaction costs have a first-order effect on liquidity premia, which is comparable to the empirical evidence. They find that, when volatility during trading periods is three times that of non-trading periods, the LPTC ratio is about 1.76, for a proportional transaction cost rate of $1 \%$.

We contribute to this body of research by considering the possibility that information about market regimes is incomplete and we find that this new ingredient can significantly amplify the magnitude of the effect of transaction costs on liquidity premia.

In our empirical analysis, we use several proxies for macro uncertainty to show that it can robustly amplify the relation between trading costs and future stock returns. We use the indices from Baker, Bloom, and Davis (2016) and Jurado, Ludvigson, and Ng (2015), as well as the VIX index. The results are consistent across the various proxies.

In a recent paper by Bali, Brown, and Tang (2017), they compute the exposure of each stock to the economic uncertainty index of Jurado, Ludvigson, and Ng (2015). They find that stocks with the lowest (negative) uncertainty beta earn 6\% extra annualized risk-adjusted return compared to the stocks with the highest (positive) uncertainty beta. They argue that their results are consistent with uncertainty-averse investors demanding extra compensation for holding stocks with negative uncertainty beta. The objective of our paper is different from theirs. We study how information uncertainty affects the relation between trading costs and future stock returns. We do not measure the exposure of each stock to the uncertainty proxies, because trading costs and uncertainty betas may be highly correlated. Instead, we use the macro uncertainty proxies directly, to classify a period as having high or low uncertainty when compared with its historical median. These aggregate 
uncertainty proxies are not related to any particular stock-level characteristic. Therefore, our regression specifications are effectively comparing the LPTC ratios between above- and belowmedian uncertainty periods.

Zhang (2006) uses the dispersion in analyst forecasts as a proxy for stock-level information uncertainty. The author shows that greater information uncertainty leads to higher average returns following good news, and relatively lower average returns following bad news. This is because investors underreact to new information. The results in our theory model also suggest that investors with incomplete information are likely to underreact to the regime shifts, as they are unobservable.

Kang, Li, and Zhang (2017) note that, even though information uncertainty and stock illiquidity are highly positively correlated measures, they have distinct effects on future stock returns. Specifically, illiquidity is positively correlated with returns, and information uncertainty is negatively correlated with returns (e.g., Diether, Malloy, and Scherbina (2002), Jiang, Lee, and Zhang (2005)). They argue that, after controlling for the positive correlation between stock illiquidity and information uncertainty, the illiquidity measure of Amihud (2002) remains an important determinant of stock returns even in the more recent decades, which have been marked by significant improvements in market liquidity and an apparent decrease in liquidity premia (e.g., Ben-Rephael, Kadan, and Wohl (2015)). We show that transaction costs are priced in the cross-section of stock returns, especially in periods of high information uncertainty at the macro level.

\section{Model: Regime Shifts and Incomplete Information}

In this section, we provide the details of our model, which accounts for regime shifts and incomplete information. The results of our model will be compared to a benchmark model with regime shifts and complete information. For brevity, we relegate the description of the benchmark model to Section A.0 of the Appendix.

\subsection{Market Structure}

We assume that an investor trades in a financial market consisting of a risky asset (stock) and a money market account. The money market account grows at a constant risk-free rate, according to the process $d B_{t}=r B_{t} d t$. The stock price process, denoted by $S_{t}$, evolves according to the following dynamics:

$$
d S_{t}=S_{t}\left[\mu\left(\varepsilon_{t}\right) d t+\sigma d w_{t}\right]
$$


where $\sigma$ is a constant volatility parameter, $\mu\left(\varepsilon_{t}\right)$ is the average return is state $\varepsilon_{t}$, and $\varepsilon_{t} \in\{1,2\}$ is a two-state Markov chain, where $\varepsilon_{t}=1$ indicates a bull market and $\varepsilon_{t}=2$ indicates a bear market. We denote by $\mu_{1}=\mu(1)$ the average return in the bull market, and $\mu_{2}=\mu(2)$ the average return in the bear market. We assume that $\mu_{1}>0>\mu_{2}$, and we use $\lambda_{1}$ and $\lambda_{2}$ to denote the switching intensities from bull to bear and from bear to bull, respectively. The variable $w_{t}$ is a standard Brownian motion independent of $\varepsilon_{t}{ }^{8}$

The investor can buy the stock at the ask price $S_{t}^{A}=(1+\theta) S_{t}$ and sell the stock at the bid price $S_{t}^{B}=(1-\alpha) S_{t}$, where $\theta \geq 0$ and $0 \leq \alpha<1$ represent the proportional transaction cost rates for purchases and sales, respectively. Trading the money market account is costless.

\subsection{Information Structure and Investor Preferences}

We extend the model of JKLL to allow for regime shifts that are unobservable to the investor. Therefore, the investor is uncertain about the state of the market she is living in at a given point in time $t$. The investor can only observe the historical price $S_{u}$ for $u \in[0, t]$, but she does not know with certainty whether the stock market is in a bull or in a bear state. In other words, the state of the market $\varepsilon_{t}$ is not observable.

In order to solve this model, we use the filtering technique proposed in Wonham (1965). Specifically, let $p_{t}=\operatorname{Prob}\left(\varepsilon_{t}=1 \mid \mathcal{S}_{t}\right)$ denote the conditional probability of $\varepsilon_{t}=1$ (bull market) given the filtration $\mathcal{S}_{t}=\Sigma\left\{S_{u}: 0 \leq u \leq t\right\}$. In a Bayesian sense, $p_{t}$ denotes the posterior distribution of the noisy stock market state. Following Wonham (1965), it can be shown that $p_{t}$ evolves according to the following process:

$$
d p_{t}=\left(-\left(\lambda_{1}+\lambda_{2}\right) p_{t}+\lambda_{2}\right) d t+\frac{\left(\mu_{1}-\mu_{2}\right) p_{t}\left(1-p_{t}\right)}{\sigma} d \widehat{w}_{t}
$$

where $\widehat{w}_{t}$ is the innovation process (see Øksendal (2003)):

$$
d \widehat{w}_{t}=\frac{d \log \left(S_{t}\right)-\left[\left(\mu_{1}-\mu_{2}\right) p_{t}+\mu_{2}-\sigma^{2} / 2\right] d t}{\sigma} .
$$

In other words, although the investor is uncertain about the state of the current market regime, she can estimate the probability of being in a bull market from observing historical prices and using

\footnotetext{
${ }^{8}$ In order to be able to use the Wonham (1965) filter in the incomplete information case, we have to assume the volatility of our risky asset returns to be constant across the market regimes.
} 
Bayesian updating. The stock price process can be re-written in terms of $\widehat{w}_{t}$, as follows:

$$
d S_{t}=S_{t}\left[\left(\mu_{1}-\mu_{2}\right) p_{t}+\mu_{2}\right] d t+S_{t} \sigma d \widehat{w}_{t}
$$

We emphasize that (1) states the true price generating process, while (4) is the investor's perceived price generating process that she uses to solve the portfolio choice problem with unobservable regime shifts.

Assume the investor holds the amount $x_{t}$ in a money market account and the amount $y_{t}$ in a stock account. In the presence of transaction costs, $x_{t}$ and $y_{t}$ evolve according to the following sub-wealth processes:

$$
d x_{t}=r x_{t} d t-(1+\theta) d L_{t}+(1-\alpha) d M_{t}
$$

and

$$
d y_{t}=\left(\left(\mu_{1}-\mu_{2}\right) p_{t}+\mu_{2}\right) y_{t} d t+\sigma y_{t} d \widehat{w}_{t}+d L_{t}-d M_{t}
$$

where $L_{t}$ and $M_{t}$ are right-continuous and non-decreasing processes, with $L_{0}=M_{0}=0$. The processes $L_{t}$ and $M_{t}$ represent cumulative dollar amounts of purchases and sales of stock, respectively. The investor's problem is then to choose the trading strategy $\left(L_{t}, M_{t}\right)$ for $0 \leq t \leq T$ to maximize the expected utility she derives from her cumulated net wealth level by the terminal date $T$ :

$$
\max _{\left(L_{t}, M_{t}\right): t \geq 0} E\left(u\left(W_{T}\right)\right)
$$

subject to the dynamic budget constraints (5) and (6), where the net wealth (i.e., after selling the long positions and covering the short positions in the stock account) is given by:

$$
W_{t}=x_{t}+(1-\alpha) y_{t}^{+}-(1+\theta) y_{t}^{-},
$$

and the utility function of the investor is of the CRRA type, taking the following form:

$$
u(W)= \begin{cases}\log (W) & \text { if } \gamma=1 \text { (log utility) } \\ \frac{W^{1-\gamma}}{1-\gamma} & \text { if } \gamma \neq 1, \gamma>0 \text { (power utility) }\end{cases}
$$

Given that investors are often limited in their ability to use excessive leverage and from taking 
large short positions, we assume that our investor is subject to the following position limits:

$$
\underline{a} \leq \frac{y_{t}}{x_{t}+y_{t}} \leq \bar{a}, \quad \forall t>0
$$

where $-\frac{1}{\theta}<\underline{a}<0$ and $1<\bar{a}<\frac{1}{\alpha}$. These constraints imply that the investor is not allowed to borrow more than $\bar{a}-1$ times her wealth or short-sell stocks of value greater than $\underline{a}$ times her wealth. When $\underline{a}=-\frac{1}{\theta}$ and $\bar{a}=\frac{1}{\alpha}$, the constraint (10) becomes the well-known solvency constraint (e.g. Davis and Norman (1990)).

We define the investor's value function as follows:

$$
\psi(x, y, p, t)=\sup _{(L, M)} E\left[u\left(W_{T}\right) \mid \mathcal{S}_{t}, x_{t}=x, y_{t}=y, p_{t}=p\right]
$$

Following Shreve and Soner (1994), it can be shown that the value function $\psi$ satisfies the following HJB equation:

$$
\left\{\begin{array}{l}
\max \left\{\psi_{t}+\mathcal{L} \psi,(1-\alpha) \psi_{x}-\psi_{y},-(1+\theta) \psi_{x}+\psi_{y}\right\}=0, \\
(1-\alpha) \psi_{x}-\psi_{y}=0 \text { on } \frac{y}{x+y}=\bar{a}, \\
(1+\theta) \psi_{x}-\psi_{y}=0 \text { on } \frac{y}{x+y}=\underline{a}, \\
\psi(x, y, p, T)=u\left(x+(1-\alpha) y^{+}-(1+\theta) y^{-}\right) .
\end{array}\right.
$$

where the differential operator is given by:

$$
\mathcal{L} \psi=\frac{\sigma^{2}}{2} y^{2} \psi_{y y}+\frac{\sigma_{p}^{2}}{2} \psi_{p p}+E_{\mu} y \psi_{y}+\sigma \sigma_{p} y \psi_{y p}+r x \psi_{x}+\left(-\left(\lambda_{1}+\lambda_{2}\right) p+\lambda_{2}\right) \psi_{p}
$$

where $E_{\mu}$ denotes the conditional expectation of the value of the (noisy) stock price drift, which is defined as follows:

$$
E_{\mu}=\left(\mu_{1}-\mu_{2}\right) p+\mu_{2}
$$

and $\sigma_{p}$ denotes the volatility of the (noisy) stock price drift, which is defined as follows:

$$
\sigma_{p}=\frac{\left(\mu_{1}-\mu_{2}\right) p(1-p)}{\sigma}
$$

We can reduce one dimension of this problem using a change of variable, by which we set 
$z=\frac{y}{x+y}$, leading to the following re-defined value function:

$$
\psi(x, y, p, t)= \begin{cases}V(z, p, t)+\log (x+y) & \text { if } \gamma=1 \text { (log utility) } \\ \frac{1}{1-\gamma}(x+y)^{(1-\gamma)} e^{(1-\gamma) V(z, p, t)} & \text { if } \gamma \neq 1, \gamma>0 \text { (power utility) }\end{cases}
$$

As a result of this change of measure, the HJB equation (12) can be simplified as follows:

$$
\left\{\begin{array}{l}
\max \left\{V_{t}+\mathcal{L}_{1} V,(\alpha z-1) V_{z}-\alpha,(1+\theta z) V_{z}-\theta\right\}=0 \\
V_{z}(\bar{a}, p, t)=\frac{\alpha}{\alpha \bar{a}-1} \\
V_{z}(\underline{a}, p, t)=\frac{\theta}{\theta \underline{a}+1} \\
V(z, p, T)=\ln \left(1-\alpha z^{+}-\theta z^{-}\right) .
\end{array}\right.
$$

where $(z, p, t) \in(\underline{a}, \bar{a}) \times[0,1] \times[0, T)$ and where the differential operator is now given by:

$$
\begin{aligned}
\mathcal{L}_{1} V= & \frac{\sigma^{2}}{2} z^{2}(1-z)^{2}\left(V_{z z}+(1-\gamma) V_{z}^{2}\right)+\frac{1}{2} \sigma_{p}^{2}\left(V_{p p}+(1-\gamma) V_{p}^{2}\right) \\
& +\sigma \sigma_{p} z(1-z)\left(V_{p z}+(1-\gamma) V_{z} V_{p}\right)+\left(-\left(\lambda_{1}+\lambda_{2}\right) p+\lambda_{2}+(1-\gamma) \sigma \sigma_{p} z\right) V_{p} \\
& +\left(E_{\mu}-r-\gamma \sigma^{2} z\right) z(1-z) V_{z}+\left(E_{\mu}-r\right) z+r-\gamma \frac{\sigma^{2}}{2} z^{2}
\end{aligned}
$$

\section{Optimal Policies and Liquidity Premia}

In this section, we start with a discussion of the calibration that we use in our baseline setup. Next, we describe the investor's optimal investment policies for the cases with and without transaction costs.

\subsection{Model Calibration}

The baseline parameter values are calibrated to the estimates of the U.S. equity market provided by Ang and Bekaert (2002). Specifically, we use the following values. The risk-free rate is set at $r=0.041$, which we calibrate from historical T-bill yields. The switching intensities from bull to bear and from bear to bull are set at $\lambda_{1}=0.2353$ and $\lambda_{2}=1.7391$, respectively. The dynamics of the stock market in the two regimes are given by $d \log S_{t}=0.1539 d t+0.1306 d w_{t}$ for the bull market, and $d \log S_{t}=-0.1546 d t+0.2438 d w_{t}$ for the bear market. From these estimates, the average return in bull and bear market states are $\mu_{1}=0.1650$ and $\mu_{2}=-0.1435$, respectively. To 
be able to apply the filtering technique in Wonham (1965), we need to assume that the volatility of stock returns $(\sigma)$ is constant across bull and bear regimes. We calculate the average volatility parameter across bull and bear regimes as follows:

$$
\sigma^{2}=\frac{\lambda_{2}}{\lambda_{1}+\lambda_{2}} 0.1306^{2}+\frac{\lambda_{1}}{\lambda_{1}+\lambda_{2}} 0.2438^{2}
$$

which yields the value $\sigma=0.1487$. Note that the average length of a single run in regime $i$ (in years) is given by $1 / \lambda_{i}$. Therefore, a bull regime in our model lasts on average 4.25 years, while a bear regime lasts on average 0.58 years (i.e., about 7 months). We also assume the baseline transaction cost rates for purchases and sales of risky asset to be equal to $0.70 \%(\alpha=\theta=0.007)$. This implies a bid-ask spread of $1.4 \%$, which is the average bid-ask spread for the sample used in Amihud and Mendelson (1986). In the baseline case, our investor's risk aversion parameter $(\gamma)$ is equal to 5, and the investment horizon is 10 years. All numerical results presented in this paper are generated from the solution of the HJB equations using finite-differences methods.

Discussion of Calibration: We allow for differences in mean returns between bull and bear regimes, but we set the volatilities in the two regimes to be equal to each other. This guarantees that the market regimes are indeed unobservable. If return volatilities were different in bull and bear regimes, the investor could perfectly identify the regime using the quadratic variation in prices. We use the point estimates of the average returns for the U.S. market documented in Ang and Bekaert (2002). They estimate a two-regime Markov switching model, and report that the mean log return is $1.2829 \%$ per month in the bull regime, and $-1.2881 \%$ per month in the bear regime. The difference between these point estimates is economically large, but they only find weak evidence that these two means are statistically different. This is because bear regimes are typically much shorter than bull regimes, which leads to a much lower number of observations for the bear markets, and larger standard errors. We use the point estimates of mean returns and we calibrate the bear regime to have a much shorter duration than the bull regime. Therefore, in our model, the bear market is also statistically less important. However, we find it to play an economically important role in the portfolio decisions of investors.

We compare our model to a benchmark model with complete information similar to the one used in JKLL. However, their calibration assumes the same mean returns but different variances across regimes. We assume different mean returns but the same variance across regimes. We use this latter calibration in both setting for comparison purposes. However, we cannot directly 
compare the quantitative results in our paper with those documented in JKLL given this difference in calibration.

\subsection{Optimal Trading Policies}

In this subsection, we start with a description of the investor's optimal policy in the absence of transaction costs, and then examine the effects associated with the introduction of trading costs.

\subsubsection{Zero Transaction Costs}

In the following theorem, we summarize the theoretical results under zero transaction costs and incomplete information. This generalizes the result in Honda (2003) to the case with position limits. ${ }^{9}$

Theorem 1. Suppose $\theta=\alpha=0$, then an investor with incomplete information maximizes power utility using the following investment policy:

$$
z^{*}(p, t)=\min \left\{\bar{a}, \max \left\{\underline{a}, \frac{E_{\mu}-r}{\sigma^{2} \gamma}+\frac{(1-\gamma) \sigma_{p}}{\sigma \gamma} V_{p}\right\}\right\}
$$

The corresponding optimal policy in the log utility case can be obtained by setting $\gamma=1$.

Proof: See Section A.1 in the Appendix.

This policy includes the expected risk premium $\left(E_{\mu}\right)$, calculated based on the conditional probability of being in a bull market $\left(p_{t}\right)$, instead of the constant risk premium used in the complete information case (see Section A.0 in the Appendix). Compared to the log-utility case, under power utility the optimal investment policy in the absence of trading costs involves an extra term $\frac{(1-\gamma) \sigma_{p}}{\sigma \gamma} V_{p}$ where $\sigma_{p}$ captures the volatility of the noisy stock price drift. This additional term can be interpreted as the hedging demand that the investor requires for the uncertainty associated with the stock price drift.

\footnotetext{
${ }^{9}$ Dai, Zhang, and Zhu (2010) and Dai, Yang, Zhang, and Zhu (2016) consider models with unobservable market parameters and transaction costs to study the trend-following trading strategy.
} 


\subsubsection{Non-Zero Transaction Costs}

In the presence of transaction costs, the state space $(z, p, t)$ is divided into three regions: the buy region $(\mathrm{BR})$, the sell region (SR), and the no-trade (NT) region. The boundary between the buy region and the no-trade region is called the buy boundary and is denoted by $z_{b}(p, t)$. The boundary between the sell region and the no-trade region is called the sell boundary, and is denoted by $z_{s}(p, t)$. The following theorem provides estimates for the locations of these boundaries.

Theorem 2. Let $\Omega=(\underline{a}, \bar{a}) \times[0,1] \times[0, T)$, then under incomplete information and non-zero transaction costs, the buy region (BR) and sell region (SR) are delimited by the following bounds:

$$
\begin{aligned}
& B R \subset\left\{(z, p, t) \in \Omega, \frac{(1+\theta) z}{1+\theta z} \leq \frac{E_{\mu}-r}{\sigma^{2} \gamma}+\frac{(1-\gamma) \sigma_{p} V_{p}(z, p, t)}{\sigma \gamma}\right\}, \\
& S R \subset\left\{(z, p, t) \in \Omega, \frac{(1-\alpha) z}{1-\alpha z} \geq \frac{E_{\mu}-r}{\sigma^{2} \gamma}+\frac{(1-\gamma) \sigma_{p} V_{p}(z, p, t)}{\sigma \gamma}\right\} .
\end{aligned}
$$

Proof: See Section A.2 in the Appendix.

For a log-utility investor (i.e., $\gamma=1$ ), we can transform the problem with incomplete information and with non-zero transaction costs into a double obstacle problem. We describe this case in Section A.3 of the Appendix.

We provide a graphical illustration of the buy and sell boundaries in Figure 1. This figure plots the optimal boundaries against the probability of being in a bull market $(p)$, at the initial time $t=0$, for three cases with different position limits. The figure shows that the buy and sell boundaries, $z_{b}(p, t)$ and $z_{s}(p, t)$ respectively, are insensitive with respect to time $t$, except when close to the terminal date $T$. Moreover, both boundaries increase with the probability $p$. This is because the estimate of average stock return increases with this probability.

[Insert Figure 1 about here]

\subsection{Effect of Transaction Costs on Liquidity Premia}

In this subsection, we examine the implications of our model for the magnitude of liquidity premia. We define the liquidity premium as the maximal amount of average return an investor is willing to forego in exchange for zero transaction costs. This is the same definition that was adopted in 
Constantinides (1986). In that seminal paper, it is shown that the liquidity premium is one order of magnitude smaller than the transaction cost rate. This is a puzzling finding, because the empirical evidence shows that transaction costs have a first-order effect on stock returns (e.g., Amihud and Mendelson (1986)). In an attempt to bridge the gap between theory and evidence, JKLL argue that the puzzling conclusion of Constantinides (1986) derives from the assumption of a constant investment opportunity set. In a regime-switching model with complete information, they show that transaction costs can indeed have a first-order effect on liquidity premia. We take one step forward by relaxing the assumption that investors have complete information about the market regimes. We find that incomplete information can further amplify the magnitude of the effect of trading costs on liquidity premia. Interestingly, the main driver of this effect in our model is distinct from that in JKKL. We provide more details below.

In our baseline case, the average return in the bear market is negative (i.e., $\mu_{2}<0$ ). An investor who is unconstrained is allowed to short sell the stock to improve her utility. Therefore, we need to account for that in the definition of the liquidity premium, which is the return an investor is willing to give up to reduce trading costs to zero. Therefore, it is subtracted from the positive average return in the bull market, and added to the negative average return in the bear market. This way, the gains from both long and short positions are reduced.

We assume that the investor holds the correct prior about the market regime at time $t=0$, that is $p_{0}=1$ if the initial state of the market is bullish and $p_{0}=0$ if the initial state is bearish. In other words, when computing the investor's utility loss due to transaction costs in the presence of incomplete information, we only consider the information uncertainty generated after time $t=0$, which can be attributed entirely to the unobservable nature of future regime shifts. This assumption helps us exclude the impact of a potentially arbitrary prior $p_{0}$ and allows for a fair comparison with the complete information case. ${ }^{10}$

Let $\psi\left(x, y, p, t ; \mu_{1}, \mu_{2}, \alpha, \theta\right)$ be the value function of an investor who only has access to incomplete information about the state of the market, as defined in (11), where $\mu_{1}$ and $\mu_{2}$ are the average returns in bull and bear markets, and $\alpha$ and $\theta$ are the transaction cost rates for purchases and sales, respectively. We define the liquidity premium as follows:

Definition 1. Let $\delta\left(p_{0}\right)$ denote the liquidity premium for an initial probability of a bull market

\footnotetext{
10 The liquidity premium in our model is a function of the initial probability $p_{0}$. We obtain quantitatively similar results when we use other values of $p_{0}$ as input, instead of assuming that the investor starts with the correct prior. For example, if we assume the prior $p_{0}$ equals the long-term mean of $p_{t}$, that is, $\frac{\lambda_{2}}{\lambda_{1}+\lambda_{2}}$, the associated LPTC ratio equals 1.251, which is still much larger than the LPTC ratio in the complete information model (0.644).
} 
$\left(p_{0}\right)$. Then $\delta\left(p_{0}\right)$ is such that it solves the following equation:

$$
\psi\left(1-z_{i}^{*}, z_{i}^{*}, p_{0}, 0 ; \mu_{1}, \mu_{2}, \alpha, \theta\right)=\psi\left(1-z_{i}^{*}, z_{i}^{*}, p_{0}, 0 ; \mu_{1}-\delta\left(p_{0}\right), \mu_{2}+\delta\left(p_{0}\right), 0,0\right),
$$

where $z_{i}^{*}=z^{*}\left(p_{0}, 0\right)$ denotes the investment policy that the investor finds optimal under zero transaction costs.

As discussed above, this definition of liquidity premium is different from that provided in JKKL, because we assume that the average return in bear markets is negative, in which case $\delta\left(p_{0}\right)$ is added to $\mu_{2}$.

The average liquidity premium across market regimes is computed as follows:

$$
\bar{\delta}=\delta(1) \frac{\lambda_{2}}{\lambda_{1}+\lambda_{2}}+\delta(0) \frac{\lambda_{1}}{\lambda_{1}+\lambda_{2}}
$$

where the weights are determined by the regime-switching intensities.

Table 1 reports the liquidity premium to transaction cost (LPTC) ratio, and the optimal notrading boundaries, in both models with complete information (Panel A) and with incomplete information (Panel B). We only provide information on the no-trading boundaries at $t=0$, since the buy and sell boundaries are insensitive with respect to time $t$, except when close to the horizon $T$. In the incomplete information case (Panel B), the boundaries vary with the probability of being in a bull market, and we show the boundaries for both $p=1$ and $p=0$. In Panel $\mathrm{A}$, the variables $Z_{b}(1,0)$ and $Z_{s}(1,0)$ denote the buy and sell boundaries in the bull market for the model with complete information, and the buy and sell boundaries for the bear market are denoted as $Z_{b}(2,0)$ and $Z_{s}(2,0)$, respectively. In Panel $\mathrm{B}$, the variables $z_{b}(1,0)$ and $z_{s}(1,0)$ denote the buy and sell boundaries in the bull market for the model with incomplete information, and the buy and sell boundaries for the bear market are denotes as $z_{b}(0,0)$ and $z_{s}(0,0)$, respectively. In the baseline case with complete information (Panel A), the buy and sell boundaries in the bull regime are flat at one, and for the bear regime they are flat at zero. This is because we assume that in the baseline model it is not possible to borrow or sell short.

\section{[Insert Table 1 about here]}

We find that, the model with incomplete information generates much larger liquidity premia than the model with complete information. For example, in the baseline case, the LPTC ratio in 
the incomplete information case is nearly $90 \%$ larger than that in the complete information case. This pattern remains similar for different variations in the values of the input parameters. This suggests that incorporating the effect of incomplete information can help us further bridge the gap between the theoretical predictions and the empirical evidence regarding the relation between trading costs and liquidity premia.

In the last three rows of both Panels $\mathrm{A}$ and $\mathrm{B}$ of Table 1, we relax the no-borrowing and noshorting constraints that we use in the baseline case. In the row called "No Short-selling," we keep the shorting constraint in place, but remove the borrowing constraint. This shifts up the buy and sell boundaries in the bull market, but keeps the boundaries unchanged for the bear market. The LPTC ratios do not improve significantly with the removal of the borrowing constraint.

In the row called "No Borrowing" we keep the borrowing constraint but remove the short-selling constraint. In this case, the buy and sell boundaries for the bull market remain unchanged, but the boundaries for the bear market shift down significantly. This increases the LPTC ratios only slightly for the incomplete information case (Panel B), but they increase by $66 \%$ for the complete information case (Panel A).

Lastly, we remove both the borrowing and shorting constraints. The results are reported in the row called "Unconstrained." The LPTC ratios remain similar to the case in which we have removed only the short-selling constraint. We conclude that the short-selling constraint plays an important role in determining the difference in magnitudes of the LPTC ratio between the two models. But, even after we remove the shorting constraint, the LPTC ratio continues to be about $30 \%$ larger in the incomplete information case, compared to the complete information case. The mechanism that drives this differential remains the same as in the baseline case.

These results translate into the following empirical hypothesis, which we test in subsection 5.1:

Hypothesis 1 (H1): The positive relation between trading costs and expected stock returns is amplified under incomplete information.

\subsubsection{Decomposition of Liquidity Premia}

What are the main sources of the additional liquidity premia that we find in the incomplete information model, compared to the benchmark model with full information? As we have discussed above, there are two main drivers of liquidity premia. First, the trading cost bill that is paid when 
rebalancing the portfolio to follow the optimal investment policy. Second, the portfolio distortions induced by trading costs, relative to the optimal position that would be chosen under zero trading costs.

We report in Table 2 some statistics generated from simulations of the investor's optimal trading policy. In the complete information case, the investor trades less frequently, but when a trade occurs, its size is large. This is because regime switches are perfectly observable, and this leads to spikes in trading volume around the switching times. This in turn commands a significant amount of transaction costs that have to be paid when regime switches occur. This is in contrast with the incomplete information case, in which the investor adjusts the portfolio more frequently, but the size of the adjustments is small. This is because she does not know whether a regime switch has occurred or not. Instead, she can only gradually update her estimate of the probability of living in the bull regime. As a result, she rebalances her portfolio less intensively and pays a smaller transaction cost bill overall. This suggests that the extra liquidity premium generated in a model with incomplete information is most likely driven by the portfolio distortions imposed by transaction costs. ${ }^{11}$

[Insert Table 2 about here]

To formally verify this intuition, we follow Dai, Li, Liu, and Wang (2016) when disentangling the part of the liquidity premium that is due to suboptimal risk exposure from the part due to the payment of transaction costs. We denote by $\delta^{0}\left(p_{0}\right)$ the liquidity premium that is due exclusively to suboptimal risk exposure. This quantity is such that it solves the following equation:

$$
\psi^{A}\left(1-z_{i}^{*}, z_{i}^{*}, p_{0}, 0 ; \mu_{1}, \mu_{2}, \alpha, \theta\right)=\psi\left(1-z_{i}^{*}, z_{i}^{*}, p_{0}, 0 ; \mu_{1}-\delta^{0}\left(p_{0}\right), \mu_{2}+\delta^{0}\left(p_{0}\right), 0,0\right)
$$

where $\psi^{A}$ is the indirect utility function of the investor when she adopts her optimal trading policy

\footnotetext{
${ }^{11}$ We highlight that, keeping everything else unchanged, the liquidity premium is expected to increase with the size of the stock allocation. This is because the larger the stock allocation, the greater the impact that trading costs have on the investor's utility. However, in Table 2, we show that the average stock allocation is lower under incomplete information compared to the case with complete information. Therefore, the higher liquidity premia that we report for the incomplete information case cannot be driven by the difference in stock allocations between the two models. Moreover, we have also calculated the investor's certainty-equivalent wealth loss (CEWL) due to transaction costs. As a fraction of the investor's initial wealth, the CEWL is $3.8 \%(4.5 \%)$ in the complete (incomplete) information case. This result further shows that the larger liquidity premia in the incomplete information model are not a result of a difference in stock allocations. Moreover, the contribution of suboptimal risk exposure to CEWL is $48.2 \%$ in the model with incomplete information, and is only $1.32 \%$ in its complete information counterpart. This confirms that, the results using CEWL are largely consistent with the decomposition we obtain for LPTC. We thank an anonymous referee for suggesting this analysis.
} 
in the presence of transaction costs, but in which it is assumed that the transaction costs are waived upon trading. This allows us to isolate the liquidity premium that is due exclusively to suboptimal risk exposure, because trading costs affect the optimal investment policy, but are not paid.

Like in the previous subsection, we report the average of these quantities, using the regime switching intensities as weights. These results are also reported in Table 1. Overall, we find that, the suboptimal risk exposure is the primary driver of the liquidity premia in the incomplete information model. For example, using our baseline calibration, the part of the LPTC ratio that is due to suboptimal risk exposure is only about $1.24 \%$ (i.e., $98.76 \%$ of the LPTC ratio is due to trading cost payments) when information is complete. However, in the incomplete information case, the part of the LPTC ratio that is due to suboptimal risk exposure is about $46.84 \%$ (i.e., $53.16 \%$ is due to payments of trading costs). We conclude that, the source of the extra liquidity premia observed for the model with incomplete information must be the suboptimal risk exposure. ${ }^{12}$

Why does the optimal investment policy deviate more from the zero-transaction-cost optimum in the incomplete information model? There are two mechanisms at work. First, when the investor only has incomplete information about the regime shifts, she needs to continuously update her estimate of the probability of living in the bull regime. From the investor's perspective, the investment opportunity set changes continuously, and her desired risk exposure also changes continuously. However, the presence of transaction costs makes it expensive to adjust risk exposure frequently.

Second, under incomplete information, the investor has an additional hedging demand for the risk of mistakenly adjusting her portfolio, which is based on her potential misidentification of the true market regime. Figure 2 illustrates how incomplete information can lead to a significant misestimation of the actual market regime. The figure shows that the investor can indeed generate large (small) estimates of the probability of being in a bull market when the regime in actually bearish (bullish). When the investor misestimates the market regime, trading the stock can generate

12 To increase the hurdle on this comparison of decompositions between the two models, we recalibrate them so that both generate an LPTC ratio with a very similar magnitude. For instance, if we increase the regime-switching intensities by multiplying $\lambda_{1}$ and $\lambda_{2}$ by 1.85 (i.e., use the recalibrated values of $\lambda_{1}=0.4353$ and $\lambda_{2}=3.2173$ ), this increases the LPTC ratio in the complete information model to 1.044, but it decreases LPTC for the incomplete information model to 1.042. By performing the decomposition using this alternative calibration, we find that the contribution of direct trading costs payments to LPTC is $98.5 \%(40.2 \%)$ in the complete (incomplete) information case. We conclude that the decomposition results remain qualitatively unchanged relative to our baseline calibration. We thank an anonymous referee for suggesting this analysis. However, this alternative calibration implies that the average length of the bear market is 0.31 years (about 2.5 months), and the length of the bull market is 2.30 years. These regime durations are much shorter than those in our baseline calibration, and may not be in line with empirical data. 
two types of costs: the cost of trading in the wrong direction and the cost of trading itself. Note that the first type of cost is absent under complete information. As a result, under incomplete information, the investor chooses a wider no-trade region to reduce the risk associated with the first type of cost. This leads to a more significant deviation from the optimal zero-cost policy.

[Insert Figure 2 about here]

These results suggest that the main driver of the large liquidity premia reported in Table 1 is not the trading expense associated with high stock turnover. This leads to another empirical hypothesis, which we test in subsection 5.2 :

Hypothesis 2 (H2): A higher degree of information incompleteness is associated with a more negative relation between trading costs and stock turnover.

\subsubsection{Comparative Statics}

Table 1 also reports the results that we obtain for alternative values of the input parameters. In this subsection, we describe this comparative statics analysis.

As the average return in the bull market $\left(\mu_{1}\right)$ increases, or as the frequency of switches from bull to bear markets $\left(\lambda_{1}\right)$ increases, both models with complete and incomplete information produce larger liquidity premia. However, we highlight that the mechanisms that generate these results are different across the two models. In the complete information model of JKLL, a larger value for $\mu_{1}$ implies a larger difference between the investor's optimal risk exposure in the bull compared to the bear market states, and a larger value of $\lambda_{1}$ implies more frequent switching from the bull to the bear market regime. Both of these factors lead to greater transaction cost bills paid for rebalancing the portfolio upon (observable) regime shifts, which then translates into larger liquidity premia. In our model with incomplete information, the liquidity premia also increase with $\mu_{1}$ because the investor is likely to revise her conditional probability measure $p_{t}$ to a larger extent in response to stock price fluctuations, hence the suboptimality of risk exposure caused by transaction costs also becomes larger, which then leads to higher liquidity premia.

To understand the implications associated with an increase in $\lambda_{1}$, recall that in our base case the average duration of the bull market is substantially longer than the duration of the bear market. 
When $\lambda_{1}$ increases, the average duration of the bull market shortens, and hence the investor is in fact subject to greater uncertainty, because the durations of bull and bear market states become more similar. The greater uncertainty together with transaction costs significantly distort the investment strategy, and the liquidity premia increase as a result.

The liquidity premia decrease in both models for an increase in the investor's risk aversion coefficient $(\gamma)$. This is because the investor's stock allocation decreases for higher risk aversion, hence the transaction costs will have a smaller impact on the investor's utility.

In our baseline model, we assume that short selling is not allowed. As a result, the investor with complete information is expected to exit the stock market and invest her entire portfolio in the money market account when the stock market is bearish, because the excess return of the bear market is negative. Therefore, if we further decrease the average return in bear markets, the optimal investment policy under complete information is expected to remain unchanged, and the liquidity premia will not be affected. However, that is not the case in a model with incomplete information. In this case, the investor can still hold positive holdings (i.e., over-invest) in the stock when the market is bearish. As the average return in the bear regime $\mu_{2}$ becomes more negative, the investor will revise her conditional estimate $p_{t}$ faster since the return differential between bull and bear regimes is larger. Therefore, the liquidity premia can increase for lower average returns in bear markets, under incomplete information. Figure 3 illustrates this effect.

\section{[Insert Figure 3 about here]}

In a model with complete information, JKLL show that the LPTC ratio decreases with the transaction cost rate. This is also the case in a model with incomplete information. Figure 4 shows that, as we decrease the transaction cost rate, the LPTC ratio increases for both models, but at a much faster rate for the model with incomplete information. For instance, for a transaction cost rate of $0.25 \%$, the LPTC ratio in the complete information case is about 0.64 , but under incomplete information, the ratio is almost triple that at about 1.90. These results suggest that when an investor is given only incomplete information about the state of the stock market regime, the magnitude of the effect of transaction costs on liquidity premia can be amplified significantly, to levels that are consistent with empirical evidence (e.g., Amihud and Mendelson (1986)).

[Insert Figure 4 about here] 
Figure 5 compares the LPTC ratios across the two models given different stock return volatility values. In the complete information case, the LPTC ratio first decreases then increases in the return volatility, but the sensitivity is small. In the incomplete information case, the LPTC ratio decreases in the return volatility at a much faster rate. The reason is that, in the incomplete information model, according to (2), a larger return volatility reduces the degree to which the investor is willing to revise her estimate of the probability of living in a bull regime. This makes the investment opportunity set vary more slowly, from the investor's perspective, and thus the cost of portfolio distortion also decreases.

[Insert Figure 5 about here]

We conclude our analysis on liquidity premia with a brief remark on the relationship between transaction costs and the market-timing ability of investors. In a fully observable regime-switching model, the investor trades more frequently and pays more transaction costs, but at the same time requires a lower liquidity premium for trading the stock. This is consistent with the idea that, an investor with complete information is better at timing the market and is therefore willing to trade more and pay the extra trading costs, as she should expect a better portfolio performance on average. In contrast, in an unobservable regime-switching model, the investor incurs lower transaction costs, but requires higher liquidity premia. This is because transaction costs can further reduce the investor's market-timing ability, which is already severely weakened by incomplete information. This can also help explain why liquidity premia are larger under incomplete information, despite the fact that it generates much lower trading volume.

\subsection{Model Extension: Information Acquisition}

This subsection proposes an extension to our model that allows for the possibility that the investor can acquire information (at a cost) to know with certainty, but only for an instant, the underlying regime she is living in.

For simplicity, we assume that the information cost is proportional to the investor's total wealth, to maintain the homogeneity of the problem. We also assume that the information cost is deducted equally from the bond and the stock positions. Let the information cost rate be $\delta$. If the investor decides to acquire information at time $\tau$, the value of her bond and stock positions exhibit a jump, 
as follows:

$$
\begin{aligned}
& x_{\tau}=(1-\delta) x_{\tau_{-}}, \\
& y_{\tau}=(1-\delta) y_{\tau_{-}} .
\end{aligned}
$$

As a result of the information acquisition, the investor can perfectly observe the underlying regime at time $\tau$, and at this instant she resets her conditional estimate of the probability of a bull market. We denote as $p_{\tau-}$ the conditional bull market probability before information acquisition, hence $p_{\tau}$ follows a binomial distribution, as follows:

$$
p_{\tau}= \begin{cases}1 & \text { with probability } p_{\tau-} \\ 0 & \text { with probability } 1-p_{\tau-}\end{cases}
$$

Let $\Theta_{[0, T]}$ be the set of all stopping times on $[0, T]$. The investor's problem is to choose a sequence of information acquisition times $0 \leq \tau_{1} \leq \tau_{2} \leq \ldots$ from $\Theta_{[0, T]}$, as well as the trading strategy $\left(L_{t}, M_{t}\right)$, for $0 \leq t \leq T$, to maximize the expected utility she derives from her net wealth level at the terminal date $T$, i.e.,

$$
\max _{\left(\tau_{i},\left(L_{t}, M_{t}\right)\right): i \geq 1, t \geq 0} E\left(u\left(W_{T}\right)\right)
$$

where $W_{T}$ is defined by equation (8) in Section 3. The rest of the model remains unchanged, and is as described in Section 3.

In this extended model, the investor's value function needs to satisfy an additional constraint, as follows:

$$
\psi(x, y, p, t) \geq p \psi((1-\delta) x,(1-\delta) y, 1, t)+(1-p) \psi((1-\delta) x,(1-\delta) y, 0, t)
$$

The above constraint can be interpreted as follows. After paying the cost, the value of the investor's bond and stock positions drop from $x$ and $y$ to $(1-\delta) x$ and $(1-\delta) y$, respectively, and she can perfectly observe the current regime. Recall that $p$ is the conditional probability of a bull market, given all historical information. Hence, with probability $p$, the investor will observe a bull market, and in this case she will reset the conditional bull market probability to 1 as a new starting point. Similarly, with probability $(1-p)$, she will observe a bear market, and in this case she 
will reset the conditional bull market probability to 0 . Therefore, the expected value of her value function, after the information acquisition decision, is given by the right hand side of condition (30) above. Because information acquisition is an option to the investor, the value function must be no less than the expected value after acquisition.

Conditional on information acquisition, the investor can rebalance her portfolio to match the true underlying regime, which reduces suboptimal risk exposure. However, the knowledge of the true regime leads to intensive rebalancing to the conditions of the revealed regime, which implies heavy trading costs at that instant, similar to what occurs in the model of JKLL at the switching times. ${ }^{13}$ As a result, this new feature is expected to increase the contribution of direct trading costs payments to the LPTC ratio, and decrease the contribution of suboptimal risk exposure, compared to our baseline model with incomplete information that does not allow for information acquisition.

In Figure 6, we plot the LPTC ratio against the information cost rate. We find that the LPTC ratio increases monotonically with the information cost. For instance, if we set the information cost to zero, the investor can have perfect information about the underlying regime at all times, which means that our extended model converges to the complete information model, and the LPTC converges to the LPTC in that model, which is 0.644 like in Table 1 (Panel A). If we increase the information cost rate to $1 \%$, the LPTC ratio increases to 0.790 . In this case, it is no longer optimal to purchase information at every instant, because that would be prohibitively costly. The investor only acquires information infrequently, and she is more likely to do so when the uncertainty about the underlying regime is highest, i.e., when $p$ is sufficiently distant from 1 or $0 .{ }^{14}$ Therefore, the suboptimal risk exposure starts to contribute more to the magnitude of the LPTC ratio when we consider a non-zero information cost rate.

\section{[Insert Figure 6 about here]}

As we continue to increase the information cost rate, we expect to reach a value for that parameter above which it is never optimal to acquire any information and have perfect knowledge about the underlying regime, because it is too costly to do so. For our baseline input values, this threshold for the information cost rate is about $1.4 \%$. For this and higher rates the investor never

\footnotetext{
13 This extended model is closest in spirit to that of Huang and Liu (2007). In their model, the investor acquires information to get more accurate estimate of the return predictor. In our extended model, the investor acquires information to know exactly the market regime at a particular instant.

${ }^{14}$ For brevity, we do not report the details of the information acquisition strategy, but the results are available from the authors upon request.
} 
finds it optimal to acquire information about the underlying regime, and the LPTC ratio converges to 1.217 and remains at that level, which is exactly what we obtain in our model with incomplete information in Table 1 (Panel B).

Overall, the finding that the LPTC increases in the information cost is consistent with our main argument that, the more incomplete is the information, the larger the impact of suboptimal risk exposure on liquidity premia. ${ }^{15}$

\section{Empirical Analysis}

In this section, we test the novel empirical implications derived from our theoretical results. First, we test Hypothesis $\mathrm{H} 1$ from subsection 4.3, which states that the higher the information uncertainty the stronger the positive relation between trading costs and future stock returns. Second, we test Hypothesis $\mathrm{H} 2$ from subsection 4.3.1, which states that the negative effect of transaction costs on stock trading volume is significantly more negative in periods of high information uncertainty. This second test aims to provide evidence that the amplification of the positive effect of trading costs on future stocks returns is not driven by the direct trading cost payments associated with portfolio rebalancing, but by the suboptimal risk exposure, which is the main channel in our theory model.

We adopt the empirical methodology of Hasbrouck (2009). We include stocks from NYSE, AMEX, and NASDAQ, for a 24-year period starting in January 1994 and ending in December 2017. We form 100 portfolios by sequentially sorting stocks based on the values of the beta estimates from the prior year (10 groups), and the effective trading cost estimates from the prior year (10 groups). Both the beta and the effective trading cost estimates are the Gibbs estimates of the basic marketfactor model in Hasbrouck (2009). ${ }^{16}$ The product of the 100 portfolios by the 12 months a year and the 24-year period used in this analysis results in a total of 28,800 observations for our final sample.

\footnotetext{
15 An important caveat is in order. We cannot guarantee that the theorems and propositions that we present in the Appendix of this paper will always hold under this more general framework with information acquisition. In addition, our assumption that we can know with certainty the market regime we are living in by paying a cost may not be realistic.

16 In his website (http://people.stern.nyu.edu/jhasbrou/Research/GibbsCurrent/gibbsCurrentIndex.html), Joel Hasbrouck provides a sample of these estimates, but only until 2009. We have extended the sample until 2017.
} 


\subsection{Incomplete Information, Trading Costs, and Future Stock Returns}

To assess the impact of information uncertainty on the relation between trading costs and future stock returns, we create an indicator variable for large values of macro uncertainty, and interact this indicator with the effective trading cost measure. We use six macro uncertainty proxies. The value of each proxy as of December of the prior year is compared with its median value in the previous 36 months. The indicator equals one if the value in December is above the historical median, and equals zero otherwise.

The uncertainty proxies that we use are as follows. We use the three-component economic policy uncertainty (EPU) measure, and the news-based measure of economic policy uncertainty (NEPU) from Baker, Bloom, and Davis (2016). We use the 12-month financial uncertainty (FINU) index, the 12-month macro uncertainty (MACU) index, and the 12-month real uncertainty (REAU) index from Jurado, Ludvigson, and Ng (2015). Lastly, we use the CBOE VIX index, which is commonly used as a proxy for uncertainty in the stock market.

Table 3 reports the results of a regression model with the following specification:

$$
\begin{aligned}
R_{i, t+1}= & \gamma_{0}+\gamma_{c} c_{i, t}+\gamma_{d} \text { AboveMedUncert }{ }_{i, t}+\gamma_{d c} \quad\left(c_{i, t} \times \text { AboveMedUncert }_{i, t}\right) \\
& +\gamma_{l r m c} \text { LRMC }_{i, t}+\gamma_{m} \beta_{i}^{m}+\gamma_{s m b} \beta_{i}^{s m b}+\gamma_{h m l} \beta_{i}^{h m l}+\epsilon_{i, t}
\end{aligned}
$$

where the dependent variable is the monthly portfolio return in the year after portfolio formation (equal-weighted across the stocks in each portfolio), AboveMedUncert is the indicator for above median macro uncertainty as of December of the prior year relative to the previous 36 months. The uncertainty proxy can be either EPU, NEPU, FINU, MACU, REAU, or VIX, as described above. The variable $L R M C$ is the $\log$ relative market capitalization (i.e., the average medianadjusted market capitalization for the stocks in each portfolio), and the variables $\beta^{m}$, $\beta^{s m b}$, and $\beta^{h m l}$ are the unconditional betas obtained from a three-factor Fama-French model estimated over the entire sample period for each portfolio. The effective trading cost measure is denoted as $c$.

[Insert Table 3 about here]

The results show a positive relation between effective trading costs and future monthly excess returns, especially (or almost exclusively) for periods with above-median uncertainty. The main coefficient of interest is on the interaction $c \times$ AboveMedUncert, which compares the liquidity 
premia between high and low uncertainty periods. In column (1), the coefficient of 0.1916 on the interaction $c \times$ Above MedEPU implies that, for a $1 \%$ increase in the effective trading cost, stocks provide an additional $0.19 \%$ monthly excess return (or $2.28 \%$ annualized) during periods defined ex-ante as having above-median EPU, compared to other periods. In column (2), we use NEPU as the proxy for uncertainty, and the additional monthly excess return for periods with aboveversus below-median uncertainty (i.e., the coefficient on the interaction $c \times$ AboveMedNEPU) is $0.51 \%$ (or $6.12 \%$ annualized), for a $1 \%$ increase in effective trading cost. The difference is even larger when using VIX as the uncertainty proxy, at about $0.53 \%$ monthly (or $6.36 \%$ annualized). If we use the uncertainty indices from Jurado, Ludvigson, and $\mathrm{Ng}$ (2015), the differences in future monthly excess returns between above- and below-median uncertainty periods are $0.32 \%$ for FINU (3.84\% annual), $0.49 \%$ for MACU (5.88\% annual), and $0.30 \%$ for REAU (3.60\% annual). These results are all statistically significant at the $5 \%$ level or better.

Overall, these results are consistent with Hypothesis $\mathrm{H} 1$ of subsection 4.3, and corroborate our theoretical argument that information uncertainty has an important amplification effect on the relation between trading costs and future stock returns.

\subsection{Incomplete Information and Trading Volume}

In general, liquidity premia can be driven not only by the trading cost charge itself (which is a function of stock trading volume) but also by the suboptimal risk exposure chosen to control the trading costs (which does not depend on trading volume). In our theory, we argue that the second channel predominates.

In the decomposition analysis of subsection 4.3.1, we show that information uncertainty weakens the contribution of trading expenses to liquidity premia. To provide evidence consistent with this theoretical implication, we estimate an empirical model using portfolio turnover as the dependent variable. ${ }^{17}$ We expect to find that, the relation between trading costs and turnover is more negative in periods of high information uncertainty, compared to periods of low uncertainty.

We report in Table 4 the results of the estimation of a regression model with the following

\footnotetext{
${ }^{17}$ We use turnover instead of trading volume, because the former is a normalized version of trading volume (i.e., the ratio of trading volume to the number of shares outstanding), which is a much less skewed variable.
} 
specification:

$$
\begin{aligned}
\text { Turn }_{i, t+1}= & \gamma_{0}+\gamma_{c} c_{i, t}+\gamma_{d} \text { AboveMedUncert }_{i, t}+\gamma_{d c} \quad\left(c_{i, t} \times \text { AboveMedUncert }_{i, t}\right) \\
& +\gamma_{s z} \operatorname{Ln}\left(\text { Size }_{i, t}+\gamma_{a} \text { Alpha }_{i, t}+\gamma_{b} \text { Beta }_{i, t}+\gamma_{i v} \text { IdioVol }_{i, t}+\gamma_{\text {div }} \text { DivYield }_{i, t}+\epsilon_{i, t}\right.
\end{aligned}
$$

where Turn is the average turnover of the stocks in each of the 100 portfolios, created following the sequential double-sort procedure described above. The indicator AboveMedUncert and the trading cost measure $(c)$ are defined as in the previous subsection. In these regressions, we control for the characteristics examined in Lo and Wang (2000). Specifically, we control for (i) the natural log of a stock's market capitalization, averaged across all stocks in a portfolio ( $\operatorname{Ln}(\operatorname{Size}))$, (ii) the intercept coefficient from the time-series regression of a stock's return on the value-weighted market return, averaged across all stocks in a portfolio (Alpha), (iii) the slope coefficient from the time-series regression of a stock's return on the value-weighted market return, averaged across all stocks in a portfolio (Beta), (iv) the residual standard deviation of the time-series regression of a stock's return on the value-weighted market return, averaged across all stocks in a portfolio (Idio Vol), and (v) the average dividend yield of the stocks in each portfolio (Div Yield). These five characteristics have been considered important determinants of stock turnover. We confirm in Table 4 that these variables are strongly (positively) related with future turnover.

The main coefficient of interest is on the interaction $c \times$ AboveMedUncert, which compares the effect of trading costs on stock turnover between high and low uncertainty periods. For four out of the six uncertainty proxies, the interaction between uncertainty and trading cost is negative and statistically significant at the $1 \%$ level, with t-statistics ranging from -4.73 to -12.83 . This suggests that stock turnover is significantly lower in above-median-uncertainty periods compared to other periods. Among the four uncertainty proxies with significant effects, EPU appears to be the strongest, with portfolio turnover being $95 \%$ lower in periods with above-median EPU, compared to below-median EPU periods. The weakest is REAU with portfolio turnover being $22 \%$ lower in periods with above-median REAU compared to periods with below-median REAU.

\section{[Insert Table 4 about here]}

The two interactions that are not significant involve the uncertainty proxies FINU and MACU from Jurado, Ludvigson, and $\mathrm{Ng}$ (2015). This indicates that the portfolio turnover is not significantly different between above- and below-median uncertainty periods when using these two 
proxies. These results are not inconsistent with our main argument. They imply that the amplification effect found in Table 3 during periods with above-median values of FINU and MACU cannot be explained by a change in turnover.

Overall, the results in Table 4 are consistent with Hypothesis H2 of subsection 4.3.1, suggesting that the main mechanism driving the higher liquidity premia in periods of above-median uncertainty is not the trading cost charge associated with portfolio turnover.

These results on turnover can shed some light on the apparently problematic findings in Hasbrouck (2009). This prior work shows that, when uncovering the positive relation between effective cost and future stock returns, the coefficient on effective cost was too large $(>1)$ to be consistent with a simple trading story. ${ }^{18}$ The author argued that "the estimated impact of effective cost on returns can only be viewed as equilibrium compensation for trading expenses." However, we show that, under incomplete information, suboptimal risk exposure can play a more important role than trading expenses in explaining liquidity premia, and this can help explain the large magnitude of the coefficients found in Hasbrouck (2009).

\section{Conclusion}

Constantinides (1986) shows that, even though transaction costs can dramatically alter the way investors trade, it does not seem to affect significantly their utility, which in turn does not command a large liquidity premium to compensate for trading costs. However, such result is in contrast with the strong relation between trading costs and future stock returns found in empirical studies, such as Amihud and Mendelson (1986).

Jang, Koo, Liu, and Loewenstein (2007) (JKLL) argue that the conclusion from Constantinides (1986) is driven by the assumption of a constant investment opportunity set. They propose a regime-switching model with complete information, and show that time-variation in investment opportunities does impact significantly the relation between trading costs and liquidity premia. However, they still cannot match the magnitude of the effect found in empirical research, meaning that this is not the full explanation for the discrepancy between theoretical and empirical findings.

In this paper, we extend the model of JKLL in a non-trivial direction. They assume that regime shifts are observable, but we argue instead that this is not a very realistic assumption,

\footnotetext{
${ }^{18}$ We did not find coefficients of such magnitude in Table 3. This can simply be because the sample used in Hasbrouck (2009) covers the period 1926-2006. In the more recent sample used for our tests, liquidity in financial markets has improved significantly.
} 
because in practice investors do not know that they are living in a bull (bear) market until they have observed (often late) that prices have been increasing (decreasing) for a period of time. We include this feature in the model, by which investors cannot observe market shifts but they can use Bayesian learning to estimate the likelihood that they live in a particular regime. We show that this simple extension of the model can have a number of significant implications. The model with incomplete information generates an amplification of the effect of trading costs on liquidity premia, to levels comparable with empirical evidence. Interestingly, the main driver of these results is very different from that in JKLL. Their result is almost entirely driven by the heavy trading cost bill that needs to be paid due to the sudden portfolio adjustments that are induced by the exogenous and fully observable regime shifts. However, in our model, investors do not observe the regime shifts, and they gradually adjust their portfolio positions as they learn from past price movements the regime they live in. As a result, they are likely to be over-invested (under-invested) in stock when the market switches from a bull to a bear (bear to bull) regime. This sluggish adjustment is further slowed down by the presence of transaction costs, and we conclude that the suboptimal risk exposure is the primary driver of the large liquidity premia generated by our model with incomplete information.

Lastly, we provide empirical evidence consistent with the novel implications of our theory model. Specifically, we show that the positive effect of trading costs on future stock returns is stronger in periods with high information uncertainty. We also show that stock turnover is not the main mechanism driving the higher liquidity premia in such periods. 


\section{References}

Amihud, Y., 2002, "Illiquidity and Stock Returns: Cross-Section and Time-Series Effects," Journal of Financial Markets, 5, 31-56.

Amihud, Y., and H. Mendelson, 1986, "Asset Pricing and the Bid-Ask Spread," Journal of Financial Economics, 17, 223-249.

Ang, A., and G. Bekaert, 2002, "International Asset Allocation With Regime Shifts," Review of Financial Studies, 15, 1137-1187.

Baker, S. R., N. Bloom, and S. J. Davis, 2016, "Measuring Economic Policy Uncertainty," Quarterly Journal of Economics, 131, 1593-1636.

Bali, T. G., S. J. Brown, and Y. Tang, 2017, "Is Economic Uncertainty Priced in the Cross-Section of Stock Returns?," Journal of Financial Economics, 126, 471-489.

Barber, B., Y.-T. Lee, Y.-J. Liu, and T. Odean, 2009, "Just How Much Do Individual Investors Lose By Trading?," Review of Financial Studies, 22, 609-632.

Ben-Rephael, A., O. Kadan, and A. Wohl, 2015, "The Diminishing Liquidity Premium," Journal of Financial and Quantitative Analysis, 50, 197-229.

Constantinides, G. M., 1986, "Capital Market Equilibrium with Transaction Costs," Journal of Political Economy, 94, 842-862.

Dai, M., H. Jin, and H. Liu, 2011, "Illiquidity, Position Limits, and Optimal Investment for Mutual Funds," Journal of Economic Theory, 146, 1598-1630.

Dai, M., P. Li, H. Liu, and Y. Wang, 2016, "Portfolio Choice with Market Closure and Implications for Liquidity Premia," Management Science, 62, 368-386.

Dai, M., Z. Yang, Q. Zhang, and Q. Zhu, 2016, "Optimal Trend Following Trading Rules," Mathematics of Operations Research, 41, 626-642.

Dai, M., and F. Yi, 2009, "Finite-Horizon Optimal Investment with Transaction Costs: A Parabolic Double Obstacle Problem," Journal of Differential Equations, 246, 1445-1469.

Dai, M., Q. Zhang, and Q. Zhu, 2010, "Trend Following Trading Under a Regime Switching Model," SIAM Journal on Financial Mathematics, 1, 780-810. 
Daniel, K., M. Grinblatt, S. Titman, and R. Wermers, 1997, "Measuring Mutual Fund Performance With Characteristic Based Benchmarks," Journal of Finance, 52, 1035-1058.

Davis, M. H. A., and A. R. Norman, 1990, "Portfolio Selection with Transaction Costs," Mathematics of Operations Research, 15, 676-713.

Diether, K., C. Malloy, and A. Scherbina, 2002, "Difference of Opinion and the Cross-Section of Stock Returns," Journal of Finance, 57, 2113-2141.

Graham, J. R., and C. R. Harvey, 1996, "Market Timing Ability and Volatility Implied in Investment Newsletters' Asset Allocation Recommendations," Journal of Financial Economics, 42, $397-421$.

Hasbrouck, J., 2009, "Trading Costs and Returns for U.S. Equities: Estimating Effective Costs from Daily Data," Journal of Finance, 64, 1445-1477.

Honda, T., 2003, "Optimal Portfolio Choice for Unobservable and Regime-switching Mean Returns," Journal of Economic Dynamics and Control, 28, 45-78.

Huang, L., and H. Liu, 2007, "Rational Inattention and Portfolio Selection," Journal of Finance, 66, 1999-2040.

Jang, B.-G., H. K. Koo, H. Liu, and M. Loewenstein, 2007, "Liquidity Premia and Transaction Costs," Journal of Finance, 62, 2330-2366.

Jiang, G., C. Lee, and Y. Zhang, 2005, "Information Uncertainty and Expected Returns," Review of Accounting Studies, 10, 185-221.

Jurado, K., S. Ludvigson, and S. Ng, 2015, "Measuring Uncertainty," American Economic Review, 105, 1177-1215.

Kacperczyk, M., and A. Seru, 2007, "Fund Manager Use of Public Information: New Evidence on Managerial Skills," Journal of Finance, 62, 485-528.

Kacperczyk, M., S. van Nieuwerburgh, and L. Veldkamp, 2014, "Time-Varying Fund Manager Skill," Journal of Finance, 69, 1455-1484.

Kang, W., N. Li, and H. Zhang, 2017, "Information Uncertainty and the Pricing of Liquidity," Working Paper, Shanghai University of Finance and Economics. 
Lei, Y., Y. Li, and J. Xu, 2019, "Two Birds, One Stone: Joint Timing of Returns and Capital Gains Taxes," forthcoming, Management Science.

Linnainmaa, J., B. Melzer, and A. Previtero, 2018, "The Misguided Beliefs and Financial Advisors," forthcoming Journal of Finance.

Liu, H., 2004, "Optimal Consumption and Investment with Transaction Costs and Multiple Risky Assets," Journal of Finance, 59, 289-338.

Liu, H., 2011, "Dynamic Portfolio Choice under Ambiguity and Regime Switching Mean Returns," Journal of Economic Dynamics and Control, 35, 623-640.

Liu, H., and M. Loewenstein, 2002, "Optimal Portfolio Selection with Transaction Costs and Finite Horizons," Review of Financial Studies, 15, 805-835.

Lo, A. W., and J. Wang, 2000, "Trading Volume: Definitions, Data Analysis, and Implications of Portfolio Theory," Review of Financial Studies, 13, 257-300.

Lynch, A. W., and S. Tan, 2010, "Multiple Risky Assets, Transaction Costs, and Return Predictability: Allocation Rules and Implications for U.S. Investors," Journal of Financial and Quantitative Analysis, 45, 1015-1053.

— , 2011, "Explaining the Magnitude of Liquidity Premia: The Roles of Return Predictability, Wealth Shocks, and State-Dependent Transaction Costs," Journal of Finance, 66, 1329-1368.

Merton, R. C., 1969, "Lifetime Portfolio Selection Under Uncertainty: The Continuous-Time Case," Review of Economics and Statistics, 51, 307-318.

— , 1971, "Optimal Consumption and Portfolio Rules in a Continuous-Time Model," Journal of Economic Theory, 3, 373-413.

Øksendal, B., 2003, Stochastic Differential Equations 6th Ed. Springer-Verlag, Berlin, New York.

Shreve, S. E., and H. M. Soner, 1994, "Optimal Investment and Consumption with Transaction Costs," Annals of Applied Probability, 4, 609-692.

Wermers, R., 2000, "Mutual Fund Performance: An Empirical Decomposition Into Stock-Picking Talent, Transactions Costs, and Expenses," Journal of Finance, 55, 1655-1703. 
Wonham, W. M., 1965, "Some Applications of Stochastic Differential Equations to Optimal Nonlinear Filtering," SIAM Journal of Control, 2, 347-369.

Xia, Y., 2001, "Learning about Predictability: The Effects of Parameter Uncertainty on Dynamic Asset Allocation," Journal of Finance, 56, 205-246.

Zhang, X. F., 2006, "Information Uncertainty and Stock Returns," Journal of Finance, 61, 105-136. 


\section{Appendix}

In this Appendix we provide a description of the benchmark model with complete information, as well as proofs and other auxiliary results.

\section{A.0. Benchmark Model: Regime Shifts and Complete Information}

In the benchmark model, the investor can observe not only stock prices but also the market regimes directly from the marketplace. In other words, the investor knows exactly what is the state of the market regime she is living in. In this case, the investor's position values $x_{t}$ and $y_{t}$ evolve according to:

$$
d x_{t}=r x_{t} d t-(1+\theta) d L_{t}+(1-\alpha) d M_{t},
$$

and

$$
d y_{t}=\mu\left(\epsilon_{t}\right) y_{t} d t+\sigma y_{t} d w_{t}+d L_{t}-d M_{t}
$$

where $L_{t}$ and $M_{t}$ also represent cumulative dollar amounts of purchases and sales of stock, respectively. The investor has the same preferences and objective, and is subject to the same position limits, as in the model with incomplete information.

In this setting, we can define the investor's value function in regime $i \in\{1,2\}$ to be

$$
\phi(x, y, i, t)=\sup _{(L, M)} E\left[u\left(W_{T}\right) \mid x_{t}=x, y_{t}=y, \varepsilon_{t}=i\right] .
$$

This value function, under some regularity conditions, can be shown to be the viscosity solution of the following HJB equation:

$$
\max \left\{\phi_{t}+\mathcal{O}^{i} \phi,(1-\alpha) \phi_{x}-\phi_{y},-(1+\theta) \phi_{x}+\phi_{y}\right\}=0, \quad i=1,2,
$$

with terminal condition:

$$
\phi(x, y, i, T)=u\left(x+(1-\alpha) y^{+}-(1+\theta) y^{-}\right),
$$

and boundary conditions:

$$
(1-\alpha) \phi_{x}-\phi_{y}=0 \text { on } \frac{y}{x+y}=\bar{a}
$$




$$
(1+\theta) \phi_{x}-\phi_{y}=0 \text { on } \frac{y}{x+y}=\underline{a}
$$

where $\mathcal{O}^{i} \phi=\frac{1}{2} \sigma^{2} y^{2} \phi_{y y}+r x \phi_{x}+\mu_{i} y \phi_{y}+\lambda_{i}(\phi(x, y, j, t)-\phi(x, y, i, t))$, for $j \neq i$.

Due to the homogeneity of the utility function, we can reduce one dimension of this problem by setting $z=\frac{y}{x+y}$ and making the following transformation

$$
\phi(x, y, i, t)= \begin{cases}\frac{1}{1-\gamma}(x+y)^{(1-\gamma)} e^{(1-\gamma) v(z, i, t)} & \text { if } \gamma \neq 1, \gamma>0 \text { (power utility) } \\ v(z, i, t)+\log (x+y) & \text { if } \gamma=1 \text { (log utility) }\end{cases}
$$

It can be verified that $v(z, i, t)$ satisfies the following PDE:

$$
\left\{\begin{array}{l}
\max \left\{v_{t}+\overline{\mathcal{O}}^{i} v+\mathcal{O}_{\gamma}^{i} v,(\alpha z-1) v_{z}-\alpha,(1+\theta z) v_{z}-\theta\right\}=0 \\
v_{z}(\bar{a}, t)=\frac{\alpha}{\alpha \bar{a}-1} \\
v_{z}(\underline{a}, t)=\frac{\theta}{\theta \underline{a}+1} \\
v(z, i, T)=\ln \left(1-\alpha z^{+}-\theta z^{-}\right)
\end{array}\right.
$$

where $(z, i, t) \in(\underline{a}, \bar{a}) \times\{1,2\} \times[0, T)$, the differential operator is given by:

$$
\overline{\mathcal{O}}^{i} v=\frac{\sigma^{2}}{2} z^{2}(1-z)^{2}\left(v_{z z}+(1-\gamma) v_{z}^{2}\right)+\left(\mu_{i}-r-\gamma \sigma^{2} z\right) z(1-z) v_{z}+\left(\mu_{i}-r\right) z+r-\gamma \frac{\sigma^{2}}{2} z^{2}
$$

and

$$
\mathcal{O}_{\gamma}^{i} v=\left\{\begin{array}{l}
\lambda_{i}(v(z, j, t)-v(z, i, t)), \quad j \neq i, \gamma=1 \text { (log utility) } \\
\frac{\lambda_{i}}{1-\gamma}\left(e^{(1-\gamma)(v(z, j, t)-v(z, i, t))}-1\right), \quad j \neq i, \gamma \neq 1, \gamma>0 \text { (power utility) }
\end{array}\right.
$$

This setting is very similar to JKLL, except that they include intertemporal consumption, and assume an exponentially distributed investment horizon. The investor in our model only cares about maximizing utility over terminal wealth, and her investment horizon is finite.

\section{Optimal Policies in the Benchmark Model}

\section{(i) Zero Transaction Costs}


The optimal investment policy under zero transaction costs and complete information is summarized in the following theorem.

Theorem A.1: Suppose $\theta=\alpha=0$, an investor with complete information maximizes power utility by keeping a constant fraction invested in stock, for each regime $i$, as follows:

$$
Z_{i}^{*}=\min \left\{\bar{a}, \max \left\{\underline{a}, \frac{\mu_{i}-r}{\sigma^{2} \gamma}\right\}\right\}
$$

The optimal policy for the logarithm utility case can be obtained by setting $\gamma=1$ in the above equation.

Proof: See Section A.1 below.

Theorem A.1 implies that, under complete information, the investor's optimal strategy in each regime is not affected by the existence of the other regime. In other words, the investor is myopic.

\section{(ii) Non-Zero Transaction Costs}

Given the concavity in $x$ and $y$ of the value function, we can characterize the optimal policy in regime $i$ by two boundaries, which split the state space $(z, t)$ into three regions: the buy region $\left(B R_{i}\right)$, the sell region $\left(S R_{i}\right)$, and the no-trade $\left(N T_{i}\right)$ region. The following theorem provides estimates for the locations of these boundaries:

Theorem A.2: Assume $Z_{i}^{*}$ is the optimal policy in regime $i$ for the case with complete information and zero transaction costs (see Theorem A.1), and assume that there exist $Z_{s}(i, t)$ and $Z_{b}(i, t)$ in each regime $i$ such that,

$$
\begin{aligned}
& B R_{i}=\left\{(z, t), z \leq Z_{b}(i, t)\right\}, \\
& S R_{i}=\left\{(z, t), z \geq Z_{s}(i, t)\right\},
\end{aligned}
$$


then we obtain the following estimates for the buy and sell boundaries, respectively:

$$
\begin{aligned}
& Z_{b}(i, t) \leq \min \left\{\max \left\{\frac{Z_{i}^{*}}{1+\theta\left(1-Z_{i}^{*}\right)}, \underline{a}\right\}, \bar{a}\right\}, \\
& Z_{s}(i, t) \geq \min \left\{\max \left\{\frac{Z_{i}^{*}}{1-\alpha\left(1-Z_{i}^{*}\right)}, \underline{a}\right\}, \bar{a}\right\} .
\end{aligned}
$$

Proof: See Section A.1 below.

Figure 7 illustrates the shape of the optimal buy and sell boundaries over the 10-year investment horizon, for both bull and bear markets, with different position limits. The results are consistent with Theorem A.2 above. Note that both buy and sell boundaries are relatively insensitive w.r.t time $t$, except when $t$ approaches the investment horizon $T$. This is due to the technical requirement that the investor must liquidate all her portfolio positions at $t=T$.

[Insert Figure 7 about here]

\section{Liquidity Premia in the Benchmark Model}

Let $\phi\left(x, y, i, t ; \mu_{1}, \mu_{2}, \alpha, \theta\right)$ be the value function of an investor in a regime-switching model with complete information, as defined in (35). The average returns in bull and bear markets are $\mu_{1}$ and $\mu_{2}$, respectively, and the transaction cost rates are $\alpha$ and $\theta$, for purchases and sales, respectively. Following JKLL, we define the liquidity premium as follows.

Definition: Let $\Delta_{i}$ denote the liquidity premium in regime $i \in\{1,2\}$ when information is complete. Then, $\Delta_{i}$ solves the following equation:

$$
\phi\left(1-Z_{i}^{*}, Z_{i}^{*}, i, 0 ; \mu_{1}, \mu_{2}, \alpha, \theta\right)=\phi\left(1-Z_{i}^{*}, Z_{i}^{*}, i, 0 ; \mu_{1}-\Delta_{i}, \mu_{2}+\Delta_{i}, 0,0\right),
$$

where $Z_{i}^{*}$ denotes the investment policy that the investor finds optimal under zero transaction costs.

The average liquidity premium across bull and bear regimes is computed as follows:

$$
\bar{\Delta}=\Delta_{1} \frac{\lambda_{2}}{\lambda_{1}+\lambda_{2}}+\Delta_{2} \frac{\lambda_{1}}{\lambda_{1}+\lambda_{2}}
$$




\section{A.1. Proofs of Theorems 1, A.1, and A.2}

When there are no transaction costs, we can use the investor's wealth $w=x+y$ as the state variable. In the benchmark model with complete information, the value function $\phi(w, i, t)$ in each regime satisfies the HJB equation

$$
\left\{\begin{array}{l}
\sup _{z_{i} \in[\underline{a}, \bar{a}]}\left[\phi_{t}+\frac{1}{2} \sigma^{2} z_{i}^{2} w^{2} \phi_{w w}+\left(r+\left(\mu_{i}-r\right) z_{i}\right) w \phi_{w}+\lambda_{i}(\phi(w, j, t)-\phi(w, i, t))\right]=0, \quad j \neq i \\
\phi(w, i, t)=u(w)
\end{array}\right.
$$

and in the regime-switching model with incomplete information, the investor's value function $\psi(w, p, t)$ follows

$$
\left\{\begin{array}{l}
\psi_{t}+\left(-\left(\lambda_{1}+\lambda_{2}\right) p+\lambda_{2}\right) \psi_{p}+\frac{\sigma_{p}^{2}}{2} \psi_{p p} \\
+\max _{z \in[\underline{a}, \bar{a}]}\left\{\frac{\sigma^{2}}{2} z^{2} w^{2} \psi_{w w}+\left[r+\left(E_{\mu}-r\right) z\right] w \psi_{w}+\sigma \sigma_{p} z w \psi_{p w}\right\}=0 \\
\psi(w, p, T)=u(w)
\end{array}\right.
$$

The solutions to the problems above have the following form,

$$
\phi(w, i, t)= \begin{cases}\log (w)+v(i, t) & \text { if } \gamma=1 \\ \frac{w^{1-\gamma}}{1-\gamma} e^{(1-\gamma) v(i, t)} & \text { if } \gamma \neq 1\end{cases}
$$

and

$$
\psi(w, p, t)= \begin{cases}\log (w)+V(p, t) & \text { if } \gamma=1 \\ \frac{w^{1-\gamma}}{1-\gamma} e^{(1-\gamma) V(p, t)} & \text { if } \gamma \neq 1\end{cases}
$$

Plugging (49) into (47) and plugging (50) into (48), we get the proofs for Theorems 1 and A.1.

\section{Proof of Theorem A.2}


We learn from (40) that,

$$
\begin{aligned}
& v_{t}+\overline{\mathcal{O}}^{i} v+\mathcal{O}_{\gamma}^{i} v \leq 0, \quad \text { in } B R_{i} \text { and } S R_{i} \\
& v_{t}+\overline{\mathcal{O}}^{i} v+\mathcal{O}_{\gamma}^{i} v=0, \quad \text { in NT. }
\end{aligned}
$$

According to the assumption, $B R_{i}$ is below no trading region in regime $i$, while $S R_{i}$ is above the no-trading region. We find $v_{t}+\overline{\mathcal{O}}^{i} v+\mathcal{O}_{\gamma}^{i} v$ is increasing (decreasing) w.r.t. $z$ in $B R_{i}\left(S R_{i}\right)$. Specifically,

$$
\begin{gathered}
\frac{\partial}{\partial z}\left(v_{t}+\overline{\mathcal{O}}^{i} v+\mathcal{O}_{\gamma}^{i} v\right) \geq 0, \text { in } B R_{i} \\
\frac{\partial}{\partial z}\left(v_{t}+\overline{\mathcal{O}}^{i} v+\mathcal{O}_{\gamma}^{i} v^{i}\right) \leq 0, \text { in } S R_{i} .
\end{gathered}
$$

It can be easily shown that

$$
\frac{\partial}{\partial z}\left(\overline{\mathcal{O}}^{i} v\right)=\widehat{\mathcal{O}}^{i} v_{z}
$$

where $\widehat{\mathcal{O}}^{i} v$ is given by

$$
\begin{aligned}
\widehat{\mathcal{O}}^{i} v= & \frac{\sigma^{2}}{2} z^{2}(1-z)^{2}\left(v_{z z}+2(1-\gamma) v v_{z}\right)+\left[\mu_{i}-r+\sigma^{2}-(2+\gamma) \sigma^{2} z\right. \\
& \left.\left.+(1-\gamma) \sigma^{2} z(1-z) v\right)\right] z(1-z) v_{z}+\left[\left(\mu_{i}-r\right)(1-2 z)\right. \\
& \left.\left.-\gamma \sigma^{2} z(2-3 z)+(1-\gamma) \sigma^{2} z(1-2 z)(1-z) v\right)\right] v-\gamma \sigma^{2} z+\mu_{i}-r .
\end{aligned}
$$

If $(z, t) \in B R_{i}$, then $v_{z}(z, i, t)=\frac{\theta}{1+\theta z}$ and,

$$
\widehat{\mathcal{O}}^{i} \frac{\theta}{1+\theta z}+\lambda_{i} e^{(1-\gamma)(v(z, j, t)-v(z, i, t))}\left(v_{z}(z, j, t)-v_{z}(z, i, t)\right) \geq 0
$$

which yields

$$
\widehat{\mathcal{O}}^{i} \frac{\theta}{1+\theta z} \geq \lambda_{i} e^{(1-\gamma)(v(z, j, t)-v(z, i, t))}\left(\frac{\theta}{1+\theta z}-v_{z}(z, j, t)\right) \geq 0
$$

After rearranging, we find,

$$
-\gamma \sigma^{2} z(1+\theta)+(1+\theta z)\left(\mu_{i}-r\right) \geq 0
$$

This inequality, together with the position limits, implies (43). In a similar way, we can obtain (44). 


\section{A.2. Proof of Theorem 2}

This proof is quite similar to that for Theorem A.2. In this case, the inequality corresponding to (52) involves the term $\frac{(1-\gamma) \sigma_{p} V_{p}}{\sigma \gamma}$ which can be positive or negative and thus cannot be dropped. Although we cannot get a similar bound for the optimal boundaries through the inequality, we can characterize the trading region by (21) and (22).

\section{A.3. Buy and Sell Boundaries for Log-Utility Investors}

Following the approach developed in Dai and Yi (2009), the optimal buy and sell boundaries for the log utility case are described in the following theorem.

Theorem A.3: $\quad$ Let $z^{*}(p)=\frac{E_{\mu}-r}{\sigma^{2}}$ be the optimal fraction invested in stock when information is incomplete and there are no transaction costs (see Theorem 1). In the log utility case, the buy and sell boundaries satisfy the following properties:

$$
\begin{aligned}
& z_{s}(p, t) \geq z_{b}(p, t), \\
& z_{b}(p, t) \leq \min \left\{\max \left\{\frac{z^{*}(p)}{1+\theta\left(1-z^{*}(p)\right)}, \underline{a}\right\}, \bar{a}\right\}, \text { if } z^{*}(p)<1+\frac{1}{\theta}, \\
& z_{b}\left(p, T^{-}\right)=\max \left\{\frac{-z^{*}(p)^{-}}{1+\theta\left(1-z^{*}(p)\right)}, \underline{a}\right\}, \\
& z_{s}(p, t) \geq \min \left\{\max \left\{\frac{z^{*}(p)}{1-\alpha\left(1-z^{*}(p)\right)}, \underline{a}\right\}, \bar{a}\right\}, \text { if } z^{*}(p)>1-\frac{1}{\alpha}, \\
& z_{s}\left(p, T^{-}\right)=\min \left\{\frac{z^{*}(p)^{+}}{1-\alpha\left(1-z^{*}(p)\right)}, \bar{a}\right\},
\end{aligned}
$$

where both boundaries are increasing w.r.t. the conditional probability of being in a bull market $(p)$.

This theorem shows that, the buy boundary is negative if the expected risk premium $\left(E_{\mu}\right)$ is negative. Similarly, the sell boundary is positive if $E_{\mu}$ is positive. Note also that, the investor is likely to increase her portfolio holdings of stock when the probability of being in a bull market $(p)$ increases. 
The proof of this theorem is as follows. Formally, we have $\frac{\partial}{\partial z} \mathcal{L}_{1} V=\mathcal{L}_{2} V_{z}$, where

$$
\begin{aligned}
\mathcal{L}_{2} u= & \frac{\sigma^{2}}{2} z^{2}(1-z)^{2} u_{z z}+\frac{1}{2} \sigma_{p}^{2} u_{p p}+\sigma \sigma_{p} z(1-z) u_{p z} \\
& +\left[E_{\mu}-r+\sigma^{2}-(2+\gamma) \sigma^{2} z+(1-\gamma) \sigma\left(\sigma z(1-z) u+\sigma_{p} V_{p}\right)\right] z(1-z) u_{z} \\
& +\left[-\left(\lambda_{1}+\lambda_{2}\right) p+\lambda_{2}+\sigma \sigma_{p}(1-z)-\gamma \sigma \sigma_{p} z+(1-\gamma) \sigma_{p}\left(\sigma_{p} u_{p}+\sigma z(1-z) u\right)\right] u_{p} \\
& +\left[\left(E_{\mu}-r\right)(1-2 z)-\gamma \sigma^{2} z(2-3 z)+(1-\gamma) \sigma(1-2 z)\left(\sigma z(1-z) u+\sigma_{p} V_{p}\right)\right] u \\
& +(1-\gamma) \sigma \sigma_{p} V_{p}-\sigma^{2} z+E_{\mu}-r .
\end{aligned}
$$

Let $\gamma=1$ in the operator $\mathcal{L}_{2} u$, then it becomes linear and all the terms including $V_{p}$ disappear. Thus we can transform Problem (17) into a double obstacle problem by the approach developed in Dai and Yi (2009). Set

$$
u(z, p, t)=V_{z}(z, p, t)
$$

it can be shown that $u$ satisfies

$$
\left\{\begin{array}{l}
\min \left\{\max \left\{-u_{t}-\mathcal{L}_{2} u, u-\frac{\theta}{1+\theta z}\right\}, u+\frac{\alpha}{1-\alpha z}\right\}=0 \\
u(z, p, T)=\chi_{\{z<0\}} \frac{\theta}{1+\theta z}+\chi_{\{z \geq 0\}} \frac{-\alpha}{1-\alpha z}
\end{array}\right.
$$

where $\mathcal{L}_{2}$ is defined by (58) with $\gamma=1$.

The complexity of the operator $\mathcal{L}_{2}$, as well as the discontinuity of the terminal condition at $z=0$, bring some difficulty to the characterization of the boundaries $z_{b}(p, t)$ and $z_{s}(p, t)$. Thanks to the Fichera criteria, we are able to deal with the problem in $\{z<0\}$ and $\{z>0\}$ independently, with no boundary condition required at $z=0$, and in both domains we can change the variable so as to simplify the differential operation.

In $\{z>0\}$ half plane, we let $w=\frac{1-z}{z}$ and $W(w, p, t)=-z^{2} u(z, p, t)+z$, then $w \in(\alpha-1, \infty)$, and it follows that

$$
\left\{\begin{array}{l}
-W_{t}-\mathcal{L}_{3} W=0, \text { if } \frac{1}{w+1+\theta}<W<\frac{1}{w+1-\alpha} \\
-W_{t}-\mathcal{L}_{3} W \leq 0, \text { if } W=\frac{1}{w+1-\alpha} \\
-W_{t}-\mathcal{L}_{3} W \geq 0, \quad \text { if } W=\frac{1}{w+1+\theta} \\
W(w, p, T)=\frac{1}{w+1-\alpha}, \quad w \in(\alpha-1, \infty)
\end{array}\right.
$$

In $\{z<0\}$ half plane, we instead let $\nu=\frac{z-1}{z}$ and $U(\nu, p, t)=z^{2} u(z, p, t)-z$, then $\nu \in(1+\theta, \infty)$, 
and it follows that

$$
\left\{\begin{array}{l}
-U_{t}-\mathcal{L}_{3} U=0, \quad \text { if } \frac{1}{\nu-1+\alpha}<U<\frac{1}{\nu-1-\theta}, \\
-U_{t}-\mathcal{L}_{3} U \geq 0, \quad \text { if } U=\frac{1}{\nu-1+\alpha} \\
-U_{t}-\mathcal{L}_{3} U \leq 0, \quad \text { if } U=\frac{1}{\nu-1-\theta} \\
U(\nu, p, T)=\frac{1}{\nu-1-\theta}, \quad \nu \in(1+\theta, \infty)
\end{array}\right.
$$

where

$$
\begin{array}{r}
\mathcal{L}_{3} W=\frac{\sigma^{2}}{2} w^{2} W_{w w}+\frac{\sigma_{p}^{2}}{2} W_{p p}-\sigma \sigma_{p} w W_{w p}+\left(2 \sigma^{2}-E_{\mu}+r\right) w W_{w} \\
-\left(\sigma \sigma_{p}+\left(\lambda_{1}+\lambda_{2}\right) p-\lambda_{2}\right) W_{p}+\left(\sigma^{2}-E_{\mu}+r\right) W
\end{array}
$$

For convenience, we define

$$
\Omega^{+}=(\alpha-1, \infty) \times[0,1], \quad \Omega^{-}=(1+\theta, \infty) \times[0,1] .
$$

We also define $S R^{+}, B R^{+}, N T^{+}$as the counterparts of $S R \cap\{z>0\}, B R \cap\{z>0\}, N T \cap\{z>0\}$, by the transformation $w=\frac{1-z}{z}$ and $S R^{-}, B R^{-}, N T^{-}$as the counterparts of $S R \cap\{z<0\}$, $B R \cap\{z<0\}, N T \cap\{z<0\}$, by the transformation $\nu=\frac{z-1}{z}$, respectively. Before we are able to prove Theorem 4, we first need to provide some useful auxiliary results.

Lemma 1. Let $W(w, p, t)$ be the solution to Problem (60), then

$$
\begin{aligned}
& W_{w}+W^{2} \leq 0 \text { in } \Omega^{+} \times[0, T], \\
& W_{p} \leq 0 \text { in } \Omega^{+} \times[0, T], \\
& W_{t} \geq 0 \text { in } \Omega^{+} \times[0, T] .
\end{aligned}
$$

Proof. The inequalities in Lemma 1 can be proved by applying comparison principle to Equation (60). For brevity we skip the details.

Lemma 2. There are two functions $w_{b}(p, t):[0,1] \times[0, T) \rightarrow[\alpha-1,+\infty]$ and $w_{s}(p, t):[0,1] \times$ $[0, T) \rightarrow[\alpha-1,+\infty]$ such that

$$
\begin{aligned}
& B R^{+}=\left\{(w, p, t) \in \Omega^{+} \times[0, T): w \geq w_{b}(p, t)\right\}, \\
& S R^{+}=\left\{(w, p, t) \in \Omega^{+} \times[0, T): w \leq w_{s}(p, t)\right\},
\end{aligned}
$$


where $w_{b}(p, t)$ and $w_{s}(p, t)$ are decreasing w.r.t. probability of a bull market $(p)$ and increasing w.r.t. time $(t)$, respectively. Moreover,

$$
\begin{aligned}
& w_{b}(p, t)>w_{s}(p, t) \text { for all } p \text { and } t \\
& w_{b}(p, t) \geq(1+\theta) \frac{1-z^{*}(p)}{z^{*}(p)}, \text { if } z^{*}(p)>0, \\
& w_{b}(p, t)=\infty, \text { if } z^{*}(p) \leq 0, \\
& w_{s}(p, t) \leq(1-\alpha) \frac{1-z^{*}(p)}{z^{*}(p)}, \text { if } z^{*}(p)>0, \\
& w_{s}\left(p, T^{-}\right)=(1-\alpha) \frac{1-z^{*}(p)}{z^{*}(p)}, \text { if } z^{*}(p)>0 \\
& w_{s}\left(p, T^{-}\right)=\infty, \text { if } z^{*}(p) \leq 0, \\
& w_{s}(p, t)>0, \text { if } \mu_{1}-r-\sigma^{2}<0 .
\end{aligned}
$$

Proof: Thanks to Lemma 2, it is not difficult to prove the existence of $w_{b}(p, t)$ and $w_{s}(p, t)$ as well as their properties. The proof is similar to that for Theorem 4.3, 4.5, 4.7 in Dai and Yi (2009). So we skip the details.

We can also obtain the corresponding lemma for $z<0$.

Lemma 3. There are two functions $\nu_{b}(p, t):[0,1] \times[0, T) \rightarrow[1+\theta, \infty]$ and $\nu_{s}(p, t):[0,1] \times[0, T) \rightarrow$ $[1+\theta,+\infty]$ such that

$$
\begin{aligned}
& B R^{-}=\left\{(\nu, p, t) \in \Omega^{-} \times[0, T]: \nu \leq \nu_{b}(p, t)\right\}, \\
& S R^{-}=\left\{(\nu, p, t) \in \Omega^{-} \times[0, T]: \nu \geq \nu_{s}(p, t)\right\} .
\end{aligned}
$$

Both $\nu_{b}(p, t)$ and $\nu_{s}(p, t)$ are increasing w.r.t. $p$ and $t$ respectively. Moreover,

$$
\begin{aligned}
& \nu_{s}(p, t)>\nu_{b}(p, t) \text { for all } p \text { and } t, \\
& \nu_{b}(p, t) \leq(1+\theta) \frac{z^{*}(p)-1}{z^{*}(p)}, \text { if } z^{*}(p)<0, \\
& \nu_{b}\left(p, T^{-}\right)=(1+\theta) \frac{z^{*}(p)-1}{z^{*}(p)}, \text { if } z^{*}(p)<0, \\
& \nu_{b}\left(p, T^{-}\right)=\infty, \text { if } z^{*}(p)>0, \\
& \nu_{s}(p, t) \geq(1-\alpha) \frac{z^{*}(p)-1}{z^{*}(p)}, \text { if } z^{*}(p)<0, \\
& \nu_{s}(p, t)=\infty, \text { if } z^{*}(p)>0 .
\end{aligned}
$$


Using Lemma 2 and Lemma 3 we can provide the proof for Theorem 3. From the two lemmas, we see that

$$
\begin{aligned}
& S R \cap\{z>0\}=\left\{(z, p, t) \in \Omega \times[0, T): z \geq \frac{1}{w_{s}(p, t)+1}, z>0\right\}, \\
& S R \cap\{z<0\}=\left\{(z, p, t) \in \Omega \times[0, T): z \geq \frac{1}{1-\nu_{s}(p, t)}, z<0\right\} .
\end{aligned}
$$

To prove the existence of $z_{s}(p, t)$, we only need to show that $\frac{1}{1-\nu_{s}(p, t)}$ and $\frac{1}{w_{s}(p, t)+1}$ cannot have non-zero value at the same point $(p, t)$. Assume there exists $\left(p_{0}, t_{0}\right)$ such that $\frac{1}{1-\nu_{s}\left(p_{0}, t_{0}\right)}<0$ and $\frac{1}{w_{s}\left(p_{0}, t_{0}\right)+1}>0$, then we have

$$
u\left(z, p_{0}, t_{0}\right)=\frac{-\alpha}{1-\alpha z}, \text { if } \frac{1}{1-\nu_{s}\left(p_{0}, t_{0}\right)}<z<0
$$

and

$$
u\left(z, p_{0}, t_{0}\right)>\frac{-\alpha}{1-\alpha z}, \text { if } 0<z<\frac{1}{w_{s}\left(p_{0}, t_{0}\right)+1} .
$$

Then,

$$
u\left(0^{-}, p_{0}, t_{0}\right)=-\alpha .
$$

In what follows, we show

$$
u\left(0^{+}, p_{0}, t_{0}\right)>-\alpha \text {. }
$$

Observe that, when $z>0$,

$$
\begin{aligned}
\frac{\partial}{\partial z}\left[(1-\alpha z)^{2}\left(u(z, p, t)+\frac{\alpha}{1-\alpha z}\right)\right] & =-\frac{\partial}{\partial z}\left[(w+1-\alpha)^{2}\left(W(w, p, t)-\frac{1}{w+1-\alpha}\right)\right] \\
& =-\frac{\partial}{\partial z}[\widetilde{W}-(w+1-\alpha)] \\
& =\frac{1}{z^{2}} \frac{\partial}{\partial w}[\widetilde{W}-(w+1-\alpha)] \leq 0 .
\end{aligned}
$$

We obtain the last inequality from the Proof of Lemma 2. According to our assumption,

$$
(1-\alpha z)^{2}\left(u(z, p, t)+\frac{\alpha}{1-\alpha z}\right)>0, \text { if } 0<z<\frac{1}{w_{s}\left(p_{0}, t_{0}\right)+1}
$$

Sending $z \rightarrow 0^{+}$, we obtain (81). 
On the other hand we define $u^{1}(p, t)=u\left(0^{+}, p, t\right), u^{2}(p, t)=u\left(0^{-}, p, t\right)$, and

$$
L_{0} u=\frac{\sigma_{p}^{2}}{2} u_{p p}+\left[-\left(\lambda_{1}+\lambda_{2}\right) p+\lambda_{2}+\sigma \sigma_{p}\right] u_{p}+\left(E_{\mu}-r\right) u
$$

and let $z \rightarrow 0^{+}$and $z \rightarrow 0^{-}$, respectively, in Problem (59), to find that

$$
\begin{cases}-u_{t}^{i}-\mathcal{L}_{0} u^{i}=E_{\mu}-r & \text { if }-\alpha<u^{i}<\theta, \\ -u_{t}^{i}-\mathcal{L}_{0} u^{i} \geq 0 & \text { if } u^{i}=-\alpha, \\ -u_{t}^{i}-\mathcal{L}_{0} u^{i} \leq 0 & \text { if } u^{i}=\theta, \\ u^{i}(p, T)= \begin{cases}\theta & \text { if } i=2, \\ -\alpha & \text { if } i=1 .\end{cases} \end{cases}
$$

Applying the maximum principle, we get $u^{1}(p, t) \leq u^{2}(p, t)$, which is a contradiction to (80) and (81). We have proven that $\frac{1}{1-\nu_{s}(p, t)}$ and $\frac{1}{w_{s}(p, t)+1}$ cannot have non-zero value at the same point and thus we are able to define $z_{s}(p, t)$ as follows,

$$
z_{s}(p, t)= \begin{cases}\frac{1}{1-\nu_{s}(p, t)} & \text { if } \frac{1}{1-\nu_{s}(p, t)}<0 \\ \frac{1}{w_{s}(p, t)+1} & \text { if } \frac{1}{w_{s}(p, t)+1}>0 \\ 0 & \text { otherwise. }\end{cases}
$$

From (78) and (79), we obtain

$$
S R=\left\{(z, p, t) \in \Omega \times[0, T]: z \geq z_{s}(p, t)\right\}
$$

Inequalities (56) follow from (68) and (76). Thus, (69) and (70) imply that

$$
z_{s}\left(p, T^{-}\right)=\frac{z^{*}(p)}{1-\alpha\left(1-z^{*}(p)\right)}, \text { if } z^{*}(p)>0
$$

and

$$
z_{s}\left(p, T^{-}\right) \leq 0, \text { if } z^{*}(p) \leq 0
$$

respectively. On the other hand, $z_{s}\left(p, T^{-}\right)<0$ contradicts $v(z, p, T)=\frac{\theta}{1+\theta z}$ if $z<0$. Thus (57) yields as a result.

Since $w_{s}(p, t)$ is decreasing and $\nu_{s}(p, t)$ is increasing w.r.t. $p$, from the definition of $z_{s}(p, t)$ we 
can easily check that $z_{s}(p, t)$ is increasing w.r.t. $p$. Observe that both $w_{s}(p, t)$ and $u_{s}(p, t)$ are increasing w.r.t. $t$, we find $z_{s}(p, t)$ is increasing w.r.t. $t$ when $z_{s}(p, t)=\frac{1}{1-\nu_{s}(p, t)}$ and decreasing when $z_{s}(p, t)=\frac{1}{w_{s}(p, t)+1}$.

Through a similar argument, we can prove the existence and properties of $z_{b}(p, t)$. Finally, (53) is easy to obtain by use of (65) and (72).

\section{A.4. Model with Time-Varying (Fully Observable) Expected Return}

In our baseline model, incomplete information leads the investor to gradually learn about the expected stock return and to adjust her portfolio accordingly. In the absence of transaction costs, the investor's desirable risk exposure changes over time as a result of the changing expected return estimate. The presence of transaction costs makes it more expensive to adjust risk exposure frequently. Thus, the continuously moving investment opportunity set (under the investor's filter) contributes to the large magnitude of the liquidity premium.

Could these results be generated using a model with stochastic (but fully observable) investment opportunities? We examine this alternative model in this subsection. ${ }^{19}$ The specification of the model with time-varying expected returns is as follows:

$$
\begin{aligned}
\frac{d S_{t}}{S_{t}} & =\left(\mu_{0}+\mu_{1} X_{t}\right) d t+\sigma d Z_{1 t}, \\
d X_{t} & =-g X_{t} d t+\nu d Z_{2 t}
\end{aligned}
$$

where $X_{t}$ is an observable return predictor and the two Brownian motions are correlated with $d Z_{1 t} d Z_{2 t}=\rho d t$.

We calculate the liquidity premia implied by this model, using various calibrations obtained from the literature. In Table 5, we report the parameter values used for each calibration, and the corresponding liquidity premia. Overall, the results show that the above model generates much smaller liquidity premia than our model. This implies that a fully observable slow-moving investment opportunity set does not necessarily generate liquidity premia with magnitudes close to the empirical findings. The investment opportunity set in model (82) and (83) does not change fast enough to generate sufficiently high liquidity premia.

\footnotetext{
${ }^{19}$ We thank an anonymous referee for suggesting this analysis.
} 
[Insert Table 5 about here]

Moreover, the results in Table 5 suggest that the main source of liquidity premia in the above model is the direct transaction cost bill, rather than the suboptimal risk exposure. The main reason for this results is that, the shocks on the stock price (i.e., $d Z_{1 t}$ ) and on the return predictor (i.e., $d Z_{2 t}$ ) are usually negatively correlated. This means that, conditional on a positive (negative) shock to the stock price, the fraction of wealth invested in the stock increases (decreases), while the stock's instantaneous expected return decreases (increases) and the investor wishes to decrease (increase) the exposure to the stock. Thus, the investor is very likely to sell (buy) after positive (negative) shocks to the stock price.

In our model with regime shifts and incomplete information, the stock price and expected return are positively correlated, and the investor is less likely to trade when the stock price moves. Thus, suboptimal risk exposure is the main driver of large liquidity premia in our model. Our empirical analysis finds support for the argument that high liquidity premia may not be driven by high turnover. 


\section{Table 1: Optimal Policies and Liquidity Premia}

This table provides information on how the optimal policies and liquidity premia change with parameter values. We show the results for both the complete information case (in the top panel) and the incomplete information case (in the bottom panel). The baseline parameters used are as follows. The risk-free rate is set at $r=0.05$. The average stock returns in the bull or bear market are calibrated to the point estimates in the basic model of Ang and Bekaert (2002), so that $\mu_{1}=0.165$ and $\mu_{2}=-0.1435$. The volatility of stock returns is set at $\sigma=0.1487$ in both markets. Assuming a constant volatility across the bull or bear market is necessary to make the underlying market state unobservable. The transition intensities from the bull to the bear market or from the bear to the bull market match the values used in JKLL, so that $\lambda_{1}=0.2353$ and $\lambda_{2}=1.7391$. This implies an average duration of 4.25 (0.58) years of the bull (bear) market. The proportional transaction costs rate is set at 0.7 percent, that is $\alpha=\theta=0.007$. The investor's risk aversion coefficient and investment horizon are set at $\gamma=5$ and $T=10$ years, respectively.

Panel A: Complete Information

\begin{tabular}{cccccccccccc}
\hline & & $Z_{b}(1,0)$ & $Z_{s}(1,0)$ & $Z_{b}(2,0)$ & $Z_{s}(2,0)$ & $\Delta_{1} / \alpha$ & $\Delta_{2} / \alpha$ & $\bar{\Delta} / \alpha$ & $\bar{\Delta}_{0} / \alpha$ & $\bar{\Delta}_{0} / \bar{\Delta} \times 100$ \\
\hline Baseline & & 1.00 & 1.00 & 0.00 & 0.00 & 0.629 & 0.758 & 0.644 & 0.008 & 1.242 \\
\hline \multirow{2}{*}{$\mu_{1}$} & $\times 1.05$ & 1.00 & 1.00 & 0.00 & 0.00 & 0.640 & 0.770 & 0.655 & 0.007 & 1.069 \\
& $\times 0.95$ & 1.00 & 1.00 & 0.00 & 0.00 & 0.616 & 0.744 & 0.631 & 0.013 & 2.060 \\
\hline \multirow{2}{*}{$\sigma$} & $\times 1.05$ & 0.98 & 1.00 & 0.00 & 0.00 & 0.617 & 0.747 & 0.633 & 0.019 & 3.002 \\
& $\times 0.95$ & 1.00 & 1.00 & 0.00 & 0.00 & 0.637 & 0.766 & 0.652 & 0.007 & 1.074 \\
\hline \multirow{2}{*}{$\lambda_{1}$} & $\times 1.05$ & 1.00 & 1.00 & 0.00 & 0.00 & 0.654 & 0.784 & 0.671 & 0.009 & 1.341 \\
& $\times 0.95$ & 1.00 & 1.00 & 0.00 & 0.00 & 0.603 & 0.732 & 0.618 & 0.008 & 1.294 \\
\hline \multirow{2}{*}{$\gamma$} & $\times 1.05$ & 1.00 & 1.00 & 0.00 & 0.00 & 0.629 & 0.758 & 0.645 & 0.010 & 1.550 \\
& $\times 0.95$ & 1.00 & 1.00 & 0.00 & 0.00 & 0.627 & 0.756 & 0.643 & 0.007 & 1.089 \\
\hline \multirow{2}{*}{$\alpha=\theta$} & $\times 1.05$ & 1.00 & 1.00 & 0.00 & 0.00 & 0.629 & 0.758 & 0.644 & 0.009 & 1.400 \\
& $\times 0.95$ & 1.00 & 1.00 & 0.00 & 0.00 & 0.629 & 0.758 & 0.644 & 0.008 & 1.242 \\
\hline No Short-selling & & 1.07 & 1.14 & 0.00 & 0.00 & 0.629 & 0.758 & 0.644 & 0.024 & 3.727 \\
No Borrowing & & 1.00 & 1.00 & -1.90 & -1.20 & 1.047 & 1.250 & 1.071 & 0.092 & 8.590 \\
Unconstrained & & 1.08 & 1.14 & -1.90 & -1.20 & 1.017 & 1.222 & 1.041 & 0.096 & 9.222 \\
\hline
\end{tabular}

Panel B: Incomplete Information

\begin{tabular}{ccccccccccc}
\hline & & $z_{b}(1,0)$ & $z_{s}(1,0)$ & $z_{b}(0,0)$ & $z_{s}(0,0)$ & $\delta_{1} / \alpha$ & $\delta_{2} / \alpha$ & $\bar{\delta} / \alpha$ & $\bar{\delta}_{0} / \alpha$ & $\bar{\delta}_{0} / \bar{\delta} \times 100$ \\
\hline Baseline & & 0.75 & 1.00 & 0.00 & 0.00 & 1.193 & 1.387 & 1.217 & 0.570 & 46.836 \\
\hline \multirow{2}{*}{$\mu_{1}$} & $\times 1.05$ & 0.81 & 1.00 & 0.00 & 0.00 & 1.259 & 1.468 & 1.284 & 0.596 & 46.417 \\
& $\times 0.95$ & 0.69 & 1.00 & 0.00 & 0.00 & 1.136 & 1.311 & 1.157 & 0.545 & 47.104 \\
\hline \multirow{2}{*}{$\sigma$} & $\times 1.05$ & 0.69 & 1.00 & 0.00 & 0.00 & 1.122 & 1.297 & 1.143 & 0.531 & 46.457 \\
& $\times 0.95$ & 0.83 & 1.00 & 0.00 & 0.00 & 1.273 & 1.482 & 1.298 & 0.604 & 46.533 \\
\hline \multirow{2}{*}{$\lambda_{1}$} & $\times 1.05$ & 0.75 & 1.00 & 0.00 & 0.00 & 1.239 & 1.433 & 1.264 & 0.593 & 46.914 \\
& $\times 0.95$ & 0.76 & 1.00 & 0.00 & 0.00 & 1.147 & 1.340 & 1.169 & 0.547 & 46.792 \\
\hline \multirow{2}{*}{$\gamma$} & $\times 1.05$ & 0.72 & 1.00 & 0.00 & 0.00 & 1.201 & 1.385 & 1.223 & 0.568 & 46.443 \\
& $\times 0.95$ & 0.80 & 1.00 & 0.00 & 0.00 & 1.187 & 1.389 & 1.211 & 0.571 & 47.151 \\
\hline \multirow{2}{*}{$\alpha=\theta$} & $\times 1.05$ & 0.75 & 1.00 & 0.00 & 0.00 & 1.167 & 1.357 & 1.190 & 0.561 & 47.143 \\
& $\times 0.95$ & 0.76 & 1.00 & 0.00 & 0.00 & 1.222 & 1.419 & 1.245 & 0.579 & 46.506 \\
\hline No Short-selling & & 0.75 & 1.12 & 0.00 & 0.00 & 1.219 & 1.387 & 1.239 & 0.572 & 46.166 \\
No Borrowing & & 0.75 & 1.00 & -1.68 & -0.68 & 1.303 & 1.814 & 1.364 & 0.658 & 48.240 \\
Unconstrained & & 0.75 & 1.12 & -1.68 & -0.68 & 1.327 & 1.814 & 1.386 & 0.660 & 47.619 \\
\hline
\end{tabular}




\section{Table 2: Simulation Results}

This table shows the results we obtained from simulating the models with complete and incomplete information. We provide information on the number of trades executed per year, during the investment period, the discounted transaction costs paid as a percentage of the initial wealth $(P V T C-I W)$, the accumulative transaction costs paid as a percentage of the terminal wealth $(F T C-T W)$, and the average proportion of total wealth invested in the stock (Stock Allocation). The values of the parameters used to produce this table are as follows. The risk-free rate is set at $r=0.041$. The average stock returns in the bull or bear market are calibrated to the point estimates in the basic model of Ang and Bekaert (2002), so that $\mu_{1}=0.165$ and $\mu_{2}=-0.1435$. The volatility of stock returns is set at $\sigma=0.1487$ in both markets. Assuming a constant volatility across the bull or bear market is necessary to make the underlying market state unobservable. The transition intensities from the bull to the bear market or from the bear to the bull market match the values used in JKLL, so that $\lambda_{1}=0.2353$ and $\lambda_{2}=1.7391$. This implies an average duration of 4.25 (0.58) years of the bull (bear) market. The proportional transaction costs rate is set at 0.7 percent, that is $\alpha=\theta=0.007$. The investor's risk aversion coefficient and investment horizon are set at $\gamma=5$ and $T=10$ years, respectively.

\begin{tabular}{lcccc}
\hline & \multicolumn{2}{c}{ Panel A: $\alpha=\theta=0.007$} & \multicolumn{2}{c}{ Panel B: $\alpha=\theta=0.015$} \\
& Complete Info. & Incomplete Info. & Complete Info. & Incomplete Info. \\
\hline Expected Number of Trade per Year & 0.507 & 9.265 & 0.518 & 6.470 \\
Expected PVTC-IW Ratio & 0.066 & 0.025 & 0.130 & 0.037 \\
Expected FTC-TW Ratio & 0.025 & 0.015 & 0.053 & 0.022 \\
Expected Stock Allocation & 0.881 & 0.674 & 0.874 & 0.654 \\
\hline
\end{tabular}




\section{Table 3: Incomplete Information and Future Stock Returns}

This table reports the results of a regression of portfolio returns on lagged trading costs, lagged information uncertainty, and their interaction. We use the GMM methodology from Hasbrouck (2009). In this analysis, we include stocks from NYSE, AMEX, and NASDAQ, for a 24-year period from January 1994 to December 2017 . We form 100 portfolios by sequentially sorting stocks based on the value of the beta estimates from the prior year (10 groups), and the effective trading cost estimates from the prior year (10 groups). The 100 portfolios times the 12 months a year times the 24 -year period results in 28,800 observations. Both beta and effective cost estimates are the Gibbs estimates of the basic market-factor model in Hasbrouck (2009). To assess the impact of information uncertainty on the relation between trading costs and stock returns, the regression includes an indicator for the periods with macro uncertainty above median, and its interaction with the effective trading cost measure. We use 6 macro uncertainty proxies measured as of December of the prior. Specifically, the value of the uncertainty proxy in December of the prior year is compared with its median value in the previous 36 months. The indicator equals one if the value in December is above the historical median, and equals zero otherwise. We use three groups of proxies for macro uncertainty: (i) the three-component economic policy uncertainty (EPU) measure, and the news-based measure of economic policy uncertainty (NEPU) from Baker, Bloom, and Davis (2016), (ii) the 12-month financial uncertainty (FINU) index, the 12-month macro uncertainty (MACU) index, and the 12-month real uncertainty (REAU) index from Jurado, Ludvigson, and Ng (2015), and (iii) the CBOE VIX index. The dependent variable is the monthly return of each portfolio in the year after formation (equal-weighted across the stocks in each portfolio). MKT Beta, SMB Beta, and HML Beta are the unconditional betas obtained from a three-factor Fama-French model estimated over the entire sample period for each portfolio. This estimation is performed concurrently to the estimation of the regression model reported in this table. $L R M C$ is the log relative market capitalization (i.e., the average median-adjusted market capitalization of the stocks in each portfolio). The effective trading cost measure is denoted as $c$. $t$-statistics are reported in parenthesis and are computed using GMM standard errors that correct for estimation error in the unconditional betas and for heteroskedasticity, and $*, * *$, and $* * *$ represent significance at the $10 \%, 5 \%$, and $1 \%$ levels, respectively.

Dependent Variable: Future Average Portfolio Returns

\begin{tabular}{|c|c|c|c|c|c|c|}
\hline & (1) & $(2)$ & $(3)$ & (4) & (5) & (6) \\
\hline c & $\begin{array}{l}0.0726 \\
(1.39)\end{array}$ & $\begin{array}{c}-0.0904 \\
(-1.53)\end{array}$ & $\begin{array}{c}0.0971^{*} \\
(1.68)\end{array}$ & $\begin{array}{c}-0.1302^{* *} \\
(-2.05)\end{array}$ & $\begin{array}{r}-0.0737 \\
(-1.34)\end{array}$ & $\begin{array}{c}-0.0241 \\
(-0.45)\end{array}$ \\
\hline c $\times$ AboveMedEPU & $\begin{array}{c}0.1916^{* *} \\
(2.01)\end{array}$ & & & & & \\
\hline AboveMedEPU & $\begin{array}{c}-0.0027^{* *} \\
(-2.53)\end{array}$ & & & & & \\
\hline $\mathrm{c} \times$ AboveMedNEPU & & $\begin{array}{l}0.5143^{* * *} \\
\quad(5.97)\end{array}$ & & & & \\
\hline AboveMedNEPU & & $\begin{array}{c}0.0008 \\
(0.74)\end{array}$ & & & & \\
\hline $\mathrm{c} \times$ AboveMedFINU & & & $\begin{array}{c}0.3247^{* * *} \\
\quad(3.76)\end{array}$ & & & \\
\hline AboveMedFINU & & & $\begin{array}{c}-0.0178^{* * *} \\
(-15.28)\end{array}$ & & & \\
\hline $\mathrm{c} \times$ AboveMedMACU & & & & $\begin{array}{l}0.4920^{* * *} \\
\quad(5.79)\end{array}$ & & \\
\hline AboveMedMACU & & & & $\begin{array}{c}-0.0128^{* * *} \\
(-12.46)\end{array}$ & & \\
\hline $\mathrm{c} \times$ AboveMedREAU & & & & & $\begin{array}{c}0.3034^{* * *} \\
(3.36)\end{array}$ & \\
\hline AboveMedREAU & & & & & $\begin{array}{c}-0.0183^{* * *} \\
(-17.82)\end{array}$ & \\
\hline $\mathrm{c} \times$ AboveMedVIX & & & & & & $\begin{array}{l}0.5340^{* * *} \\
\quad(5.71)\end{array}$ \\
\hline AboveMedVIX & & & & & & $\begin{array}{c}-0.0123^{* * *} \\
(-10.41)\end{array}$ \\
\hline LRMC & $\begin{array}{c}-0.0015^{* * *} \\
(-2.82)\end{array}$ & $\begin{array}{c}-0.0013^{* *} \\
(-2.52)\end{array}$ & $\begin{array}{c}-0.0013^{* *} \\
(-2.54)\end{array}$ & $\begin{array}{c}-0.0019^{* * *} \\
(-3.86)\end{array}$ & $\begin{array}{c}-0.0022^{* * *} \\
(-4.36)\end{array}$ & $\begin{array}{c}-0.0013^{* * *} \\
(-2.62)\end{array}$ \\
\hline MKT Beta & $\begin{array}{c}0.0004 \\
(0.14)\end{array}$ & $\begin{array}{c}-0.0000 \\
(-0.01)\end{array}$ & $\begin{array}{c}0.0009 \\
(0.32)\end{array}$ & $\begin{array}{c}0.0015 \\
(0.54)\end{array}$ & $\begin{array}{c}0.0019 \\
(0.72)\end{array}$ & $\begin{array}{l}0.0004 \\
(0.14)\end{array}$ \\
\hline SMB Beta & $\begin{array}{c}-0.0104^{* * *} \\
(-3.33)\end{array}$ & $\begin{array}{c}-0.0100^{* * *} \\
(-3.21)\end{array}$ & $\begin{array}{c}-0.0117^{* * *} \\
(-3.77)\end{array}$ & $\begin{array}{c}-0.0115^{* * *} \\
(-3.68)\end{array}$ & $\begin{array}{c}-0.0115^{* * *} \\
(-3.71)\end{array}$ & $\begin{array}{c}-0.0110^{* * *} \\
(-3.52)\end{array}$ \\
\hline HML Beta & $\begin{array}{l}0.0113^{* * * *} \\
(2.90)\end{array}$ & $\begin{array}{l}0.0112^{* * *} \\
(2.87)\end{array}$ & $\begin{array}{c}0.0107^{* * * *} \\
(2.75)\end{array}$ & $\begin{array}{c}0.0118^{* * * *} \\
(3.03)\end{array}$ & $\begin{array}{c}0.0123^{* * * *} \\
(3.15)\end{array}$ & $\begin{array}{c}0.0108^{* * *} \\
(2.76)\end{array}$ \\
\hline Intercept & $\begin{array}{c}-0.0006 \\
(-0.20)\end{array}$ & $\begin{array}{c}-0.0025 \\
(-0.86)\end{array}$ & $\begin{array}{c}0.0052^{*} \\
(1.84)\end{array}$ & $\begin{array}{c}0.0050^{*} \\
(1.76)\end{array}$ & $\begin{array}{c}0.0079^{* * *} \\
(2.80)\end{array}$ & $\begin{array}{c}0.0032 \\
(1.13)\end{array}$ \\
\hline Observations & 28,800 & 28,800 & 28,800 & 28,800 & 28,800 & 28,800 \\
\hline
\end{tabular}




\section{Table 4: Incomplete Information and Turnover}

This table reports the results of a regression of portfolio turnover on lagged trading costs, lagged information uncertainty, and their interaction. We use the same methodology and sample as in Table 3. We include an indicator for periods with macro uncertainty above median, and its interaction with the effective trading cost measure. We also use the 6 macro uncertainty proxies used in Table 3. The dependent variable is the monthly turnover (i.e., the ratio of trading volume to number of shares outstanding) of each portfolio in the year after formation (equal-weighted across the stocks in each portfolio). We control for several stock characteristics that are important determinants of stock turnover, following Section 4.1 of ?. Specifically, we control for (i) the natural log of a stock's market capitalization, averaged across all stocks in a portfolio $(\operatorname{Ln}($ Size $))$, (ii) the intercept coefficient from the time-series regression of a stock's return on the value-weighted market return, averaged across all stocks in a portfolio (Alpha), (iii) the slope coefficient from the time-series regression of a stock's return on the value-weighted market return, averaged across all stocks in a portfolio (Beta), (iv) the residual standard deviation of the time-series regression of a stock's return on the value-weighted market return, averaged across all stocks in a portfolio (Idio Vol), and ( $\mathrm{v}$ ) the average dividend yield of the stocks in each portfolio (DivYield). $t$-statistics are reported in parenthesis and are computed using GMM standard errors that correct for estimation error in the unconditional betas and for heteroskedasticity, and $*, * *$, and $* * *$ represent significance at the $10 \%, 5 \%$, and $1 \%$ levels, respectively.

Dependent Variable: Future Average Portfolio Turnover

\begin{tabular}{|c|c|c|c|c|c|c|}
\hline & (1) & $(2)$ & (3) & (4) & $(5)$ & (6) \\
\hline $\mathrm{c}$ & $\begin{array}{c}-1.4031^{* * *} \\
(-14.06)\end{array}$ & $\begin{array}{c}-1.3524^{* * *} \\
(-13.68)\end{array}$ & $\begin{array}{c}-1.7808^{* * *} \\
(-14.52)\end{array}$ & $\begin{array}{c}-1.6603^{* * *} \\
(-13.16)\end{array}$ & $\begin{array}{c}-1.5293^{* * *} \\
(-13.31)\end{array}$ & $\begin{array}{c}-1.5942^{* * *} \\
(-14.37)\end{array}$ \\
\hline $\mathrm{c} \times$ AboveMedEPU & $\begin{array}{c}-1.3310^{* * *} \\
(-12.83)\end{array}$ & & & & & \\
\hline AboveMedEPU & $\begin{array}{c}0.0192^{* * *} \\
(18.79)\end{array}$ & & & & & \\
\hline $\mathrm{c} \times$ AboveMedNEPU & & $\begin{array}{c}-1.0409^{* * *} \\
(-11.92)\end{array}$ & & & & \\
\hline AboveMedNEPU & & $\begin{array}{c}0.0127^{* * *} \\
(12.57)\end{array}$ & & & & \\
\hline $\mathrm{c} \times$ AboveMedFINU & & & $\begin{array}{c}-0.0765 \\
(-1.03)\end{array}$ & & & \\
\hline AboveMedFINU & & & $\begin{array}{c}0.0127^{* * *} \\
(10.16)\end{array}$ & & & \\
\hline $\mathrm{c} \times$ AboveMedMACU & & & & $\begin{array}{c}-0.0866 \\
(-1.20)\end{array}$ & & \\
\hline AboveMedMACU & & & & $\begin{array}{c}0.0168^{* * *} \\
(17.41)\end{array}$ & & \\
\hline $\mathrm{c} \times$ AboveMedREAU & & & & & $\begin{array}{c}-0.3431^{* * *} \\
(-4.73)\end{array}$ & \\
\hline AboveMedREAU & & & & & $\begin{array}{c}0.0159^{* * *} \\
(15.96)\end{array}$ & \\
\hline $\mathrm{c} \times$ AboveMedVIX & & & & & & $\begin{array}{c}-0.5221^{* * *} \\
\quad(-6.70)\end{array}$ \\
\hline AboveMedVIX & & & & & & $\begin{array}{c}0.0150^{* * *} \\
(12.02)\end{array}$ \\
\hline $\operatorname{Ln}($ Size $)$ & $\begin{array}{c}0.0217^{* * *} \\
(43.38)\end{array}$ & $\begin{array}{c}0.0221^{* * *} \\
(45.92)\end{array}$ & $\begin{array}{c}0.0234^{* * *} \\
(48.65)\end{array}$ & $\begin{array}{c}0.0223^{* * *} \\
(47.48)\end{array}$ & $\begin{array}{c}0.0224^{* * *} \\
(48.26)\end{array}$ & $\begin{array}{c}0.0230^{* * *} \\
(48.64)\end{array}$ \\
\hline Alpha & $\begin{array}{c}0.7222^{* *} \\
(1.98)\end{array}$ & $\begin{array}{c}0.5738 \\
(1.62)\end{array}$ & $\begin{array}{c}0.3470 \\
(1.01)\end{array}$ & $\begin{array}{c}0.3924 \\
(1.13)\end{array}$ & $\begin{array}{c}0.3780 \\
(1.09)\end{array}$ & $\begin{array}{c}0.3950 \\
(1.15)\end{array}$ \\
\hline Beta & $\begin{array}{l}0.1078^{* * *} \\
(15.74)\end{array}$ & $\begin{array}{l}0.1055^{* * *} \\
(15.81)\end{array}$ & $\begin{array}{c}0.0992^{* * *} \\
(15.36)\end{array}$ & $\begin{array}{l}0.1031^{* * *} \\
(15.72)\end{array}$ & $\begin{array}{l}0.1027^{* * *} \\
(15.70)\end{array}$ & $\begin{array}{l}0.1008^{* * *} \\
\quad(15.57)\end{array}$ \\
\hline IdioVol & $\begin{array}{c}0.6504^{* * *} \\
(26.85)\end{array}$ & $\begin{array}{c}0.6378^{* * *} \\
(26.55)\end{array}$ & $\begin{array}{c}0.6677^{* * *} \\
(26.33)\end{array}$ & $\begin{array}{c}0.6175^{* * *} \\
(25.95)\end{array}$ & $\begin{array}{c}0.6187^{* * * *} \\
(25.90)\end{array}$ & $\begin{array}{c}0.6569^{* * *} \\
(26.42)\end{array}$ \\
\hline DivYield & $\begin{array}{l}1.7148^{* * *} \\
(6.30)\end{array}$ & $\begin{array}{l}1.7248^{* * *} \\
(6.34)\end{array}$ & $\begin{array}{l}1.6992^{* * *} \\
(6.28)\end{array}$ & $\begin{array}{c}1.7166^{* * *} \\
(6.33)\end{array}$ & $\begin{array}{l}1.7182^{* * *} \\
\quad(6.33)\end{array}$ & $\begin{array}{l}1.7036^{* * *} \\
\quad(6.29)\end{array}$ \\
\hline Intercept & $\begin{array}{l}-0.3452^{* * *} \\
(-49.51)\end{array}$ & $\begin{array}{c}-0.3446^{* * *} \\
(-49.63)\end{array}$ & $\begin{array}{c}-0.3614^{* * *} \\
(-47.63)\end{array}$ & $\begin{array}{l}-0.3485^{* * *} \\
(-50.02)\end{array}$ & $\begin{array}{c}-0.3491^{* * *} \\
(-51.09)\end{array}$ & $\begin{array}{c}-0.3572^{* * *} \\
(-48.59)\end{array}$ \\
\hline Observations & 28,800 & 28,800 & 28,800 & 28,800 & 28,800 & 28,800 \\
\hline
\end{tabular}


Table 5: Liquidity Premia for Models with Return Predictability

This table reports various calibrations of a model with return predictability, represented by equations (82) and (83), and the liquidity premia generated under each calibration. In this model, the stock price drift is stochastic but fully observable. The specifications from Xia (2001) and Lei, Li and Xu (2019) use the dividend yield as the return predictor, but Xia (2001) adjusts for inflation. the specification from Huang and Liu (2007) uses the consumption-wealth ratio as the return predictor.

Xia (2001) Huang and Liu (2007) Lei, Li, and Xu (2019)

\section{Parameter Values}

$\begin{array}{lccc}\mu_{0} & 0.091 & 0.025 & 0.116 \\ \mu_{1} & 4.540 & 2.040 & 4.397 \\ \sigma & 0.144 & 0.080 & 0.147 \\ g & 0.190 & 0.180 & 0.141 \\ \nu & 0.006 & 0.007 & 0.005 \\ \rho & -0.930 & -0.620 & -0.895 \\ r & 0.034 & 0.003 & 0.048\end{array}$

\section{Liquidity Premia}

LPTC

LPTC (Suboptimal Exposure)
0.221

0.014
0.353

0.093
0.193

0.005 

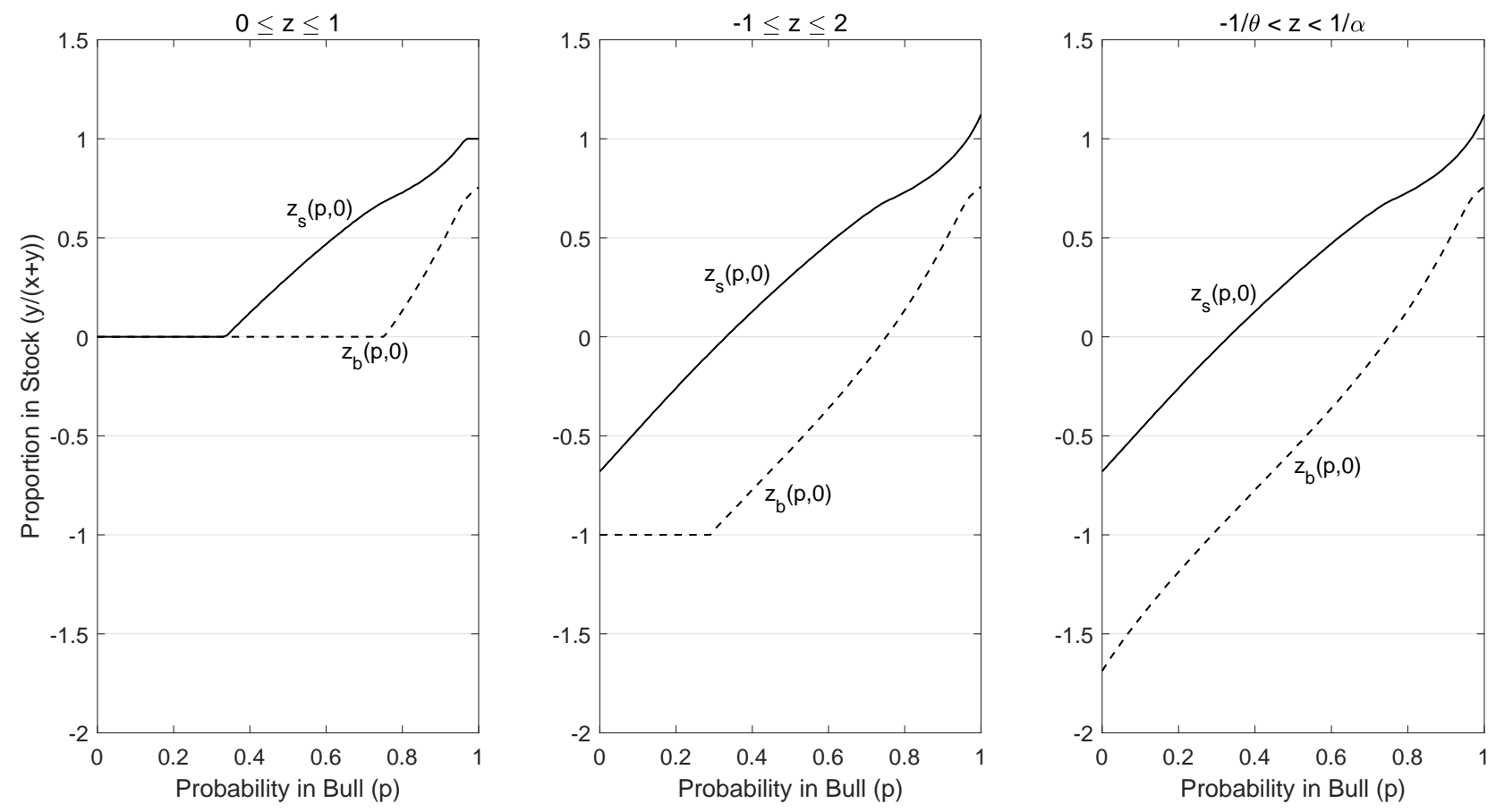

Figure 1: Optimal Policy for Incomplete Information and Trading Costs

This figure shows the no-trade boundaries, at initial time $t=0$, as functions of the conditional probability of being in a bull market (i.e., $p$ ). This figure includes three graphs. The graph on the left illustrates the case with strict no-leverage and no-short-sale constraint (i.e., $0 \leq \frac{y_{t}}{x_{t}+y_{t}} \leq 1$ )the middle graph represents the case with a position limit of $-1 \leq \frac{y_{t}}{x_{t}+y_{t}} \leq 2$, and the graph on the right is the case with only the solvency constraint (i.e., $-1 / \theta \leq \frac{y_{t}}{x_{t}+y_{t}} \leq 1 / \alpha$ ). The values of the parameters used to obtain these boundaries are as follows. The risk-free rate is set at $r=0.041$. The average stock returns in the bull or bear market are calibrated to the point estimates in the basic model of Ang and Bekaert (2002), so that $\mu_{1}=0.165$ and $\mu_{2}=-0.1435$. The volatility of stock returns is set at $\sigma=0.1487$ in both markets. Assuming a constant volatility across the bull or bear market is necessary to make the underlying market state unobservable. The transition intensities from the bull to the bear market or from the bear to the bull market match the values used in JKLL, so that $\lambda_{1}=0.2353$ and $\lambda_{2}=1.7391$. This implies an average duration of 4.25 (0.58) years of the bull (bear) market. The proportional transaction costs rate is set at $0.7 \%$, that is $\alpha=\theta=0.007$. The investor's risk aversion coefficient and investment horizon are set at $\gamma=5$ and $T=10$ years, respectively. 

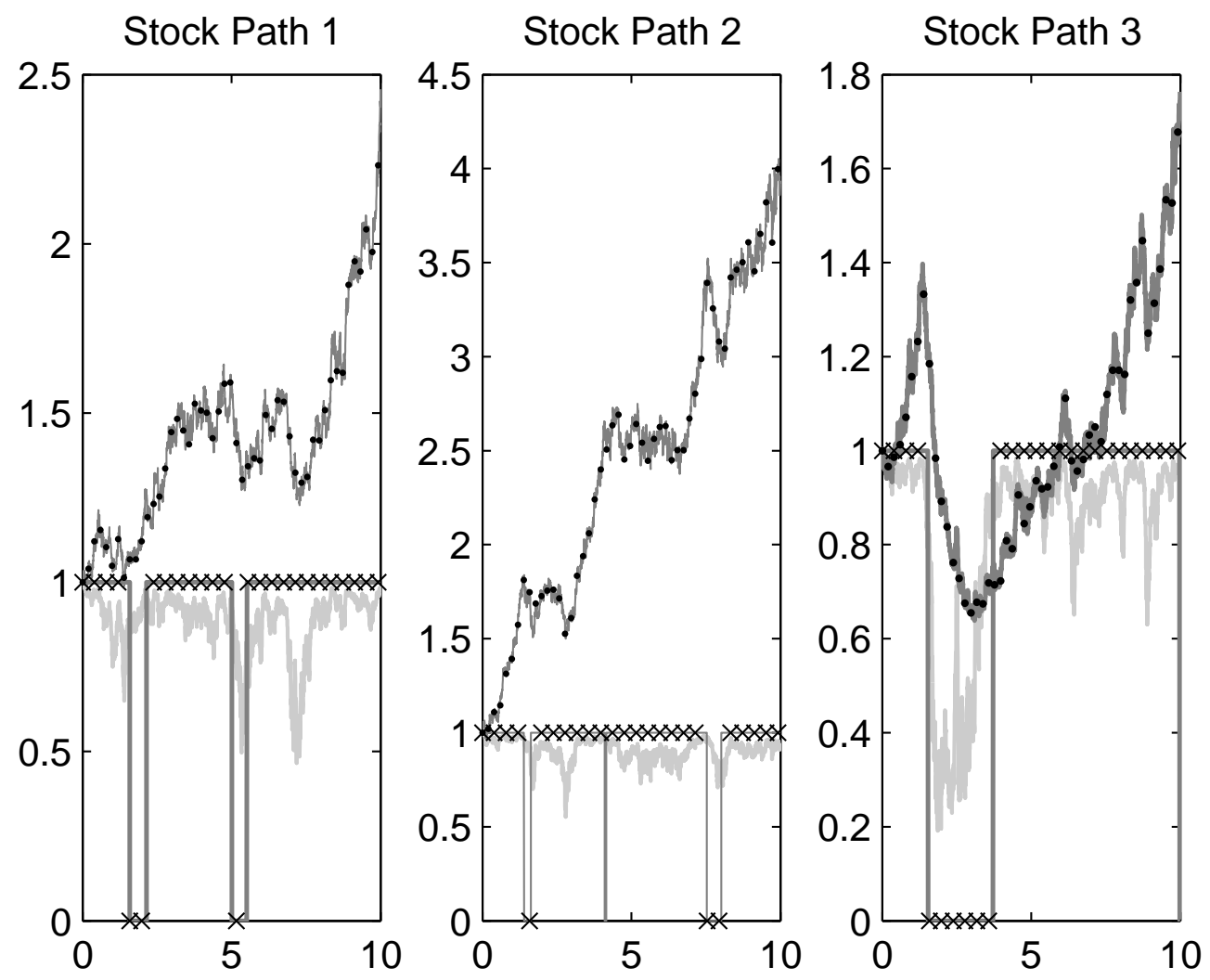

Figure 2: Probability of Bull Market and Actual Regime

This figure illustrates, for three examples of stock price paths, how the investor's estimates of the probability of being in a bull market can fail to identify the actual market regimes. The line with the marker $\bullet$ represents the stock price path for a 10-year period. The line with marker $\times$ shows the actual market regime in place (a value of one corresponds to a bull market, and a value of zero represents a bear market). The solid grey line with no marker indicates the probability estimated by the investor of being in a bull market. The values of the parameters used to obtain these figures are as follows. The average stock returns in the bull or bear market are calibrated to the point estimates in the basic model of Ang and Bekaert (2002), so that $\mu_{1}=0.165$ and $\mu_{2}=-0.1435$. The volatility of stock returns is set at $\sigma=0.1487$ in both markets. Assuming a constant volatility across the bull or bear market is necessary to make the underlying market state unobservable. The transition intensities from bull to bear and from bear to bull markets match the values used in JKLL, so that $\lambda_{1}=0.2353$ and $\lambda_{2}=1.7391$, respectively. This implies an average duration of 4.25 (0.58) years of the bull (bear) market. 


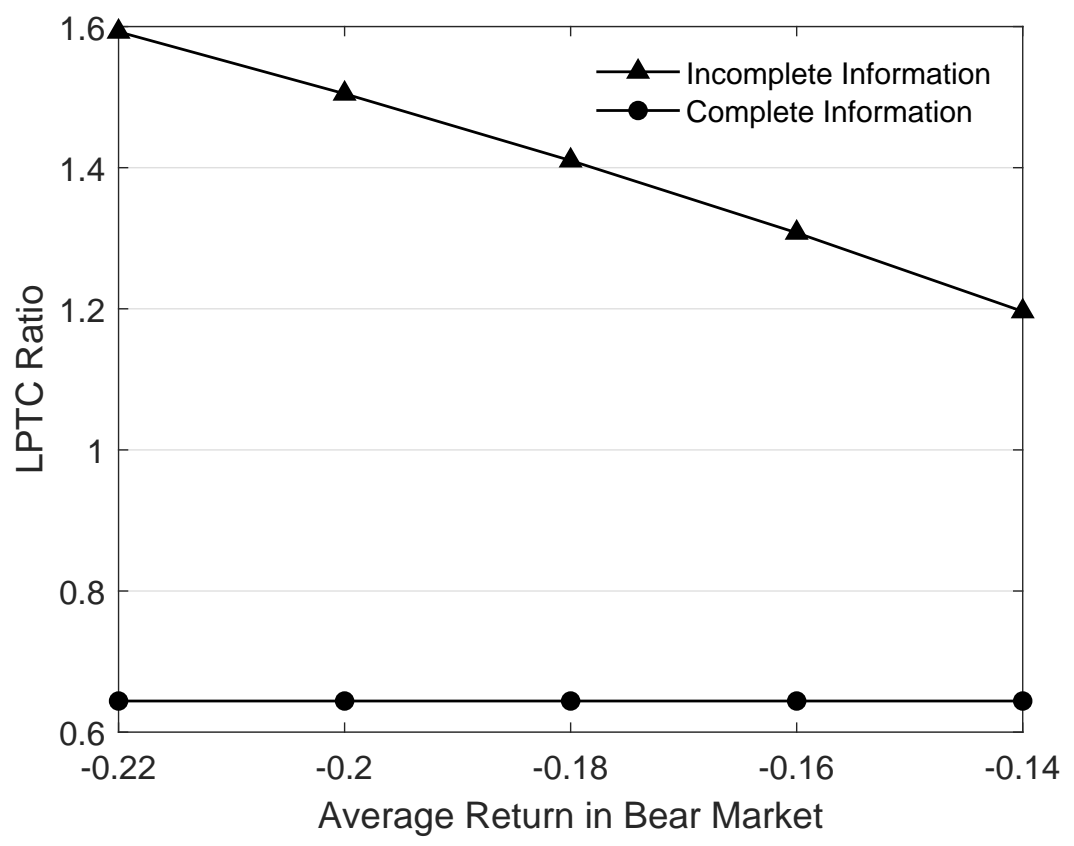

Figure 3: LPTC Ratio and Average Return in Bear Market

This figure shows how the liquidity premium to transaction cost (LPTC) ratios change with changes in the average stock return in bear markets, in both models with complete and incomplete information. The baseline parameter values used to obtain this figure are as follows. The risk-free rate is set at $r=0.041$. The average stock return in the bull market is calibrated to the point estimate in the basic model of Ang and Bekaert (2002), so that $\mu_{1}=0.165$. The average return in the bear market ranges from -0.22 to -0.14 . The volatility of stock returns is set at $\sigma=0.1487$ in both markets. Assuming a constant volatility across the bull or bear market is necessary to make the underlying market state unobservable. The transition intensities from bull to bear and from bear to bull markets match the values used in JKLL, so that $\lambda_{1}=0.2353$ and $\lambda_{2}=1.7391$, respectively. This implies an average duration of 4.25 (0.58) years of the bull (bear) market. The proportional transaction costs rate is set at $0.7 \%$, that is $\alpha=\theta=0.007$. The investor's risk aversion coefficient and investment horizon are set at $\gamma=5$ and $T=10$ years, respectively. The investor is not allowed to short the stock or borrow, that is, the values of the position limits are $\underline{a}=0$ and $\bar{a}=1$. 


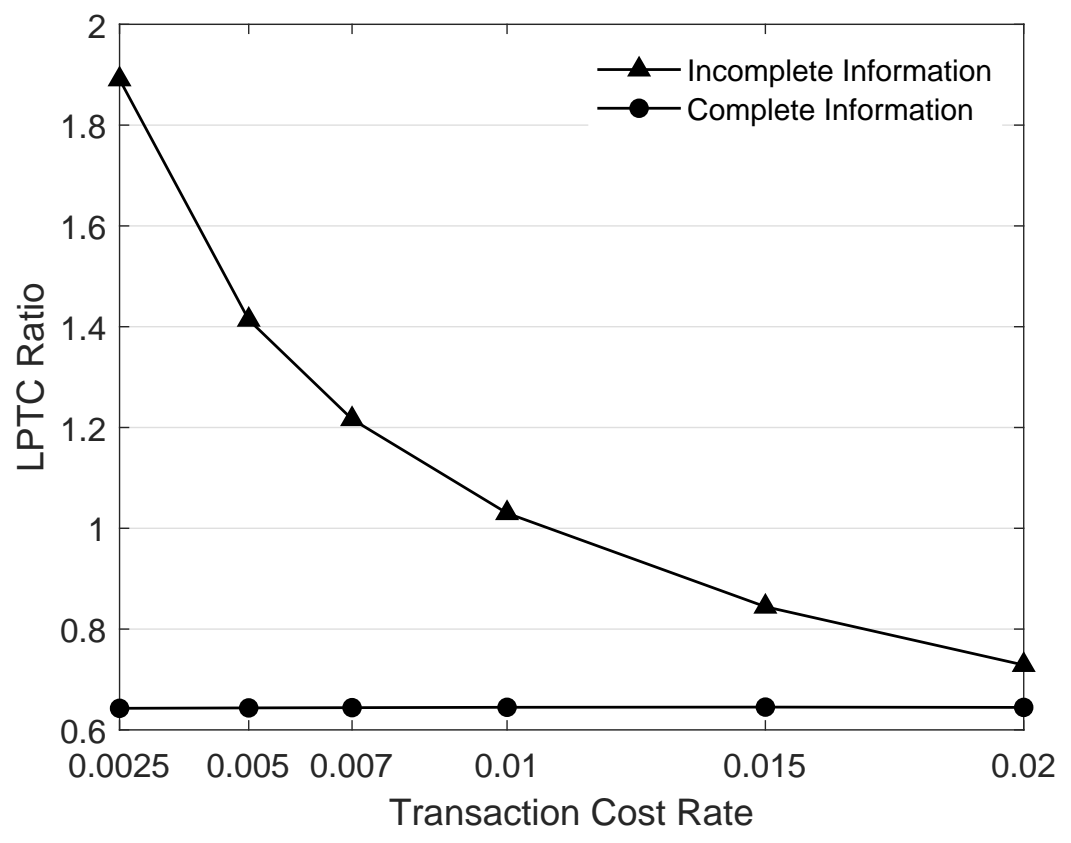

Figure 4: LPTC Ratio and Transaction Cost Rate

This figure shows how the liquidity premium to transaction cost (LPTC) ratio changes with changes in the (one way) transaction cost rate, that is $\alpha=\theta$, in both models with complete and incomplete information. The baseline parameter values used to obtain this figure are as follows. The risk-free rate is set at $r=0.041$. The average stock returns in the bull or bear market are calibrated to the point estimates in the basic model of Ang and Bekaert (2002), so that $\mu_{1}=0.165$ and $\mu_{2}=-0.1435$. The volatility of stock returns is set at $\sigma=0.1487$ in both markets. Assuming a constant volatility across the bull or bear market is necessary to make the underlying market state unobservable. The transition intensities from bull to bear and from bear to bull markets match the values used in JKLL, so that $\lambda_{1}=0.2353$ and $\lambda_{2}=1.7391$, respectively. This implies an average duration of 4.25 (0.58) years of the bull (bear) market. The investor's risk aversion coefficient and investment horizon are set at $\gamma=5$ and $T=10$ years, respectively. The investor is not allowed to short the stock or borrow, that is, the values of the position limits are $\underline{a}=0$ and $\bar{a}=1$. 


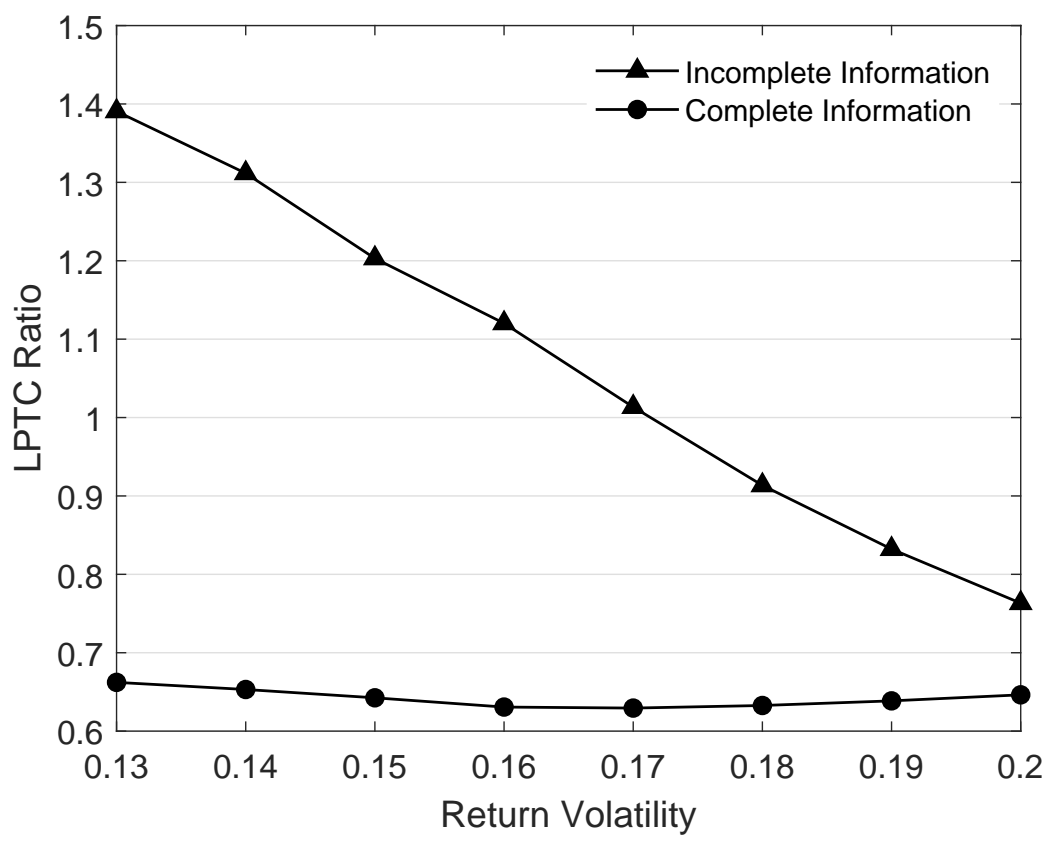

Figure 5: LPTC Ratio and Volatility

This figure shows how the liquidity premium to transaction cost (LPTC) ratios change as a function of volatility, for both the complete and incomplete information models. The values of the parameters used to obtain this figure are as follows. The risk-free rate is set at $r=0.041$. The average stock returns in the bull or bear market are calibrated to the point estimates in the basic model of Ang and Bekaert (2002), so that $\mu_{1}=0.165$ and $\mu_{2}=-0.1435$. The volatility of stock returns ranges from 0.13 to 0.20 in both markets. Assuming a constant volatility across the bull or bear market is necessary to make the underlying market state unobservable. The transition intensities from bull to bear and from bear to bull markets match the values used in JKLL, so that $\lambda_{1}=0.2353$ and $\lambda_{2}=1.7391$, respectively. This implies an average duration of $4.25(0.58)$ years of the bull (bear) market. The proportional transaction costs rate is set at $0.7 \%$, that is $\alpha=\theta=0.007$. The investor's risk aversion coefficient and investment horizon are set at $\gamma=5$ and $T=10$ years, respectively. The investor is not allowed to short the stock or borrow, that is, the values of the position limits are $\underline{a}=0$ and $\bar{a}=1$. 


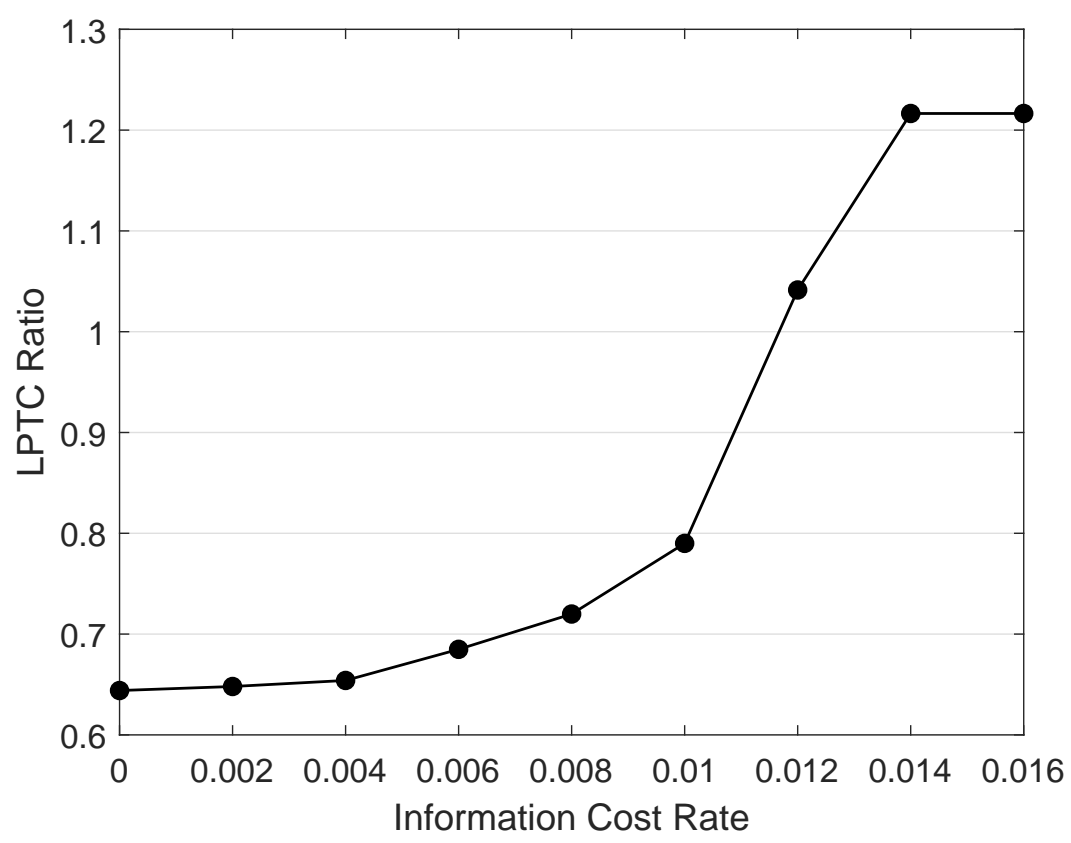

Figure 6: LPTC Ratio and Information Cost Rate

This figure shows how the liquidity premium to transaction cost (LPTC) ratios change as a function of the information cost rate. The information cost rate ranges from $0 \%$ to $1.6 \%$. The values of the remaining parameters used to obtain this figure are as in the baseline model. The risk-free rate is set at $r=0.041$. The average stock returns in the bull or bear market are calibrated to the point estimates in the basic model of Ang and Bekaert (2002), so that $\mu_{1}=0.165$ and $\mu_{2}=-0.1435$. The volatility of stock returns is set at $\sigma=0.1487$ in both markets. The transition intensities from bull to bear and from bear to bull markets match the values used in JKLL, so that $\lambda_{1}=0.2353$ and $\lambda_{2}=1.7391$, respectively. This implies an average duration of 4.25 (0.58) years of the bull (bear) market. The proportional transaction costs rate is set at $0.7 \%$, that is $\alpha=\theta=0.007$. The investor's risk aversion coefficient and investment horizon are set at $\gamma=5$ and $T=10$ years, respectively. The investor is not allowed to short the stock or borrow, that is, the values of the position limits are $\underline{a}=0$ and $\bar{a}=1$. 

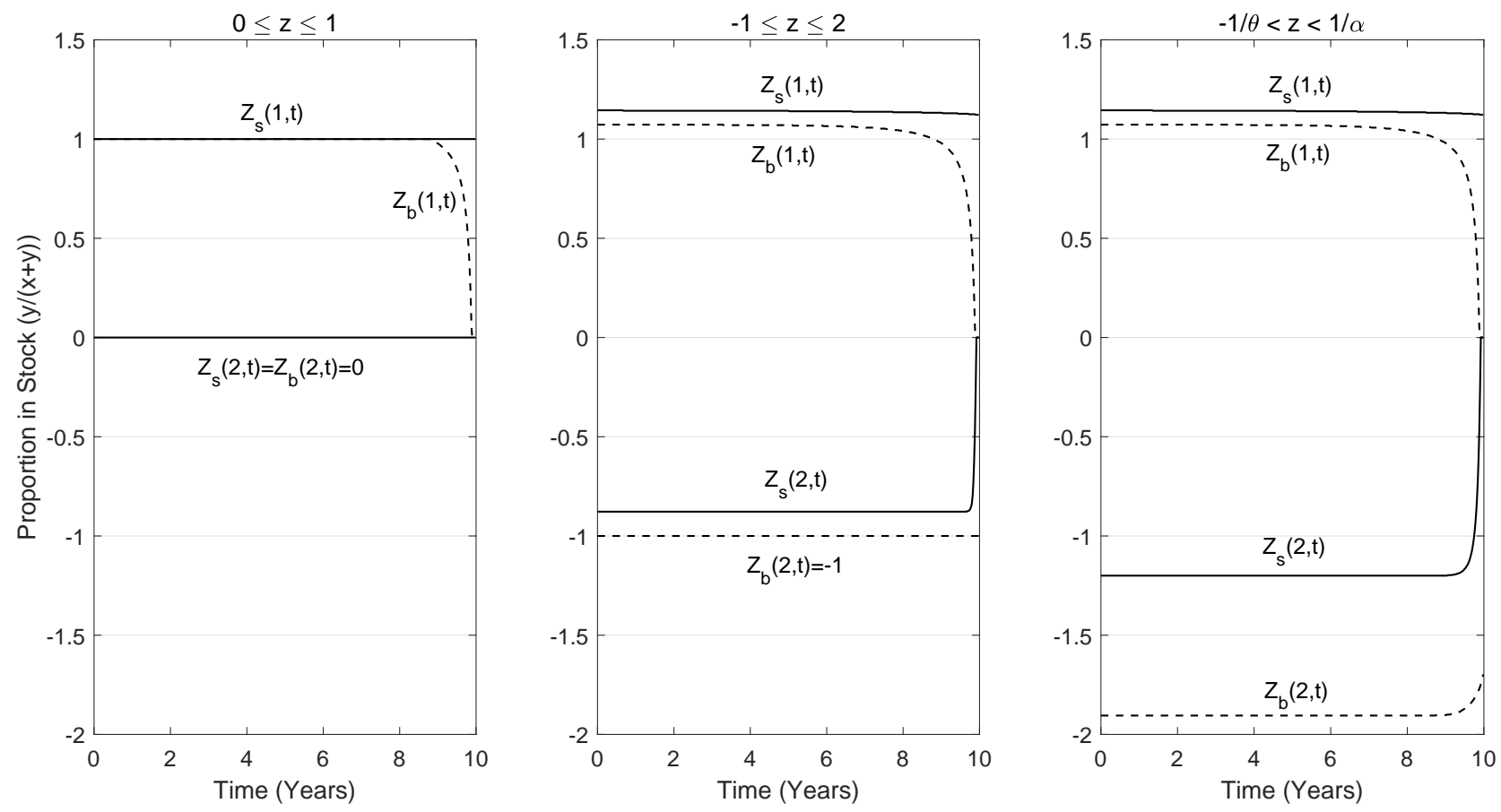

Figure 7: Optimal Policy for Complete Information and Trading Costs

This figure illustrates the shape of the no-trade boundaries over the entire investment period, for both the bull $(Z(1, t))$ and the bear markets $(Z(2, t))$, for the case of an investor who has access to complete information about the state of the market regimes, and who is subject to transaction costs. This figure includes three graphs. The graph on the left illustrates the case with strict no-leverage and no-short-sale constraint (i.e., $\left.0 \leq \frac{y_{t}}{x_{t}+y_{t}} \leq 1\right)$ the middle graph represents the case with a position limit of $-1 \leq \frac{y_{t}}{x_{t}+y_{t}} \leq 2$, and the graph on the right is the case with only the solvency constraint (i.e., $-1 / \theta \leq \frac{y_{t}}{x_{t}+y_{t}} \leq 1 / \alpha$ ). The values of the parameters used to obtain these boundaries, are as follows. The risk-free rate is set at $r=0.041$. The average stock returns in the bull or bear market are calibrated to the point estimates in the basic model of Ang and Bekaert (2002), so that $\mu_{1}=0.165$ and $\mu_{2}=-0.1435$. The volatility of stock returns is set at $\sigma=0.1487$ in both markets. Assuming a constant volatility across the bull or bear market is necessary to make the underlying market state unobservable in the incomplete information model. For comparison purposes, we calibrate the complete information model with a constant volatility across the two market regimes. The transition intensities from bull to bear and from bear to bull markets match the values used in JKLL, so that $\lambda_{1}=0.2353$ and $\lambda_{2}=1.7391$, respectively. This implies an average duration of $4.25(0.58)$ years of the bull (bear) market. The proportional transaction costs rate is set at $0.7 \%$, that is $\alpha=\theta=0.007$. The investor's risk aversion coefficient and investment horizon are set at $\gamma=5$ and $T=10$ years, respectively. 